TRANSACTIONS OF THE

AMERICAN MATHEMATICAL SOCIETY

Volume 352, Number 1, Pages 457-475

S 0002-9947(99)02319-3

Article electronically published on July 26, 1999

\title{
FINITE GROUPS OF MATRICES OVER GROUP RINGS
}

\author{
GERALD CLIFF AND ALFRED WEISS
}

\begin{abstract}
We investigate certain finite subgroups $\Gamma$ of $G L_{n}(\mathbf{Z} \Pi)$, where $\Pi$ is a finite nilpotent group. Such a group $\Gamma$ gives rise to a $\mathbf{Z}[\Gamma \times \Pi]$-module; we study the characters of these modules to limit the structure of $\Gamma$. We also exhibit some exotic subgroups $\Gamma$.
\end{abstract}

\section{INTRODUCTION}

Let $\Pi$ be a finite group. We set

$$
S G L_{n}(\mathbf{Z \Pi})=\text { ker aug }: G L_{n}(\mathbf{Z} \Pi) \rightarrow G L_{n}(\mathbf{Z}),
$$

where aug is the usual augmentation map applied to each entry of $G L_{n}(\mathbf{Z \Pi})$.

Suppose that $\alpha$ is a homomorphism from a finite group $\Gamma$ to $S G L_{n}(\mathbf{Z \Pi})$. We shall investigate the following problem.

Problem 0. Do there exist group homomorphisms $\sigma_{i}: \Gamma \rightarrow \Pi, i=1,2, \ldots, n$, and an element $x \in G L_{n}(\mathbf{Q} \Pi)$ such that $x^{-1} \alpha(\gamma) x=\operatorname{diag}\left(\sigma_{i}(\gamma)\right), \gamma \in \Gamma$ ?

This is analogous to a conjecture of Zassenhaus, who was interested in units of ZI of augmentation 1, i.e. $S G L_{1}(\mathbf{Z} \Pi)$. Problem 0 is related to results on units of group rings, as shown in a special case in $[\mathrm{MRSW}]$. There is a positive answer to Problem 0 if $\Pi$ is a $p$-group [WAn] or if $n=1$ and $\Pi$ is nilpotent [WCr].

Given finite groups $\Gamma$ and $\Pi$, set

$$
G=\Gamma \times \Pi, \quad N=1 \times \Pi .
$$

A homomorphism $\alpha: \Gamma \rightarrow S G L_{n}(\mathbf{Z \Pi})$ gives rise to a double action $\mathbf{Z} G$-module $M(\alpha)$, defined as follows: as abelian group, $M(\alpha)$ is equal to the column vectors $\mathbf{Z} \Pi^{n}$, and the $G$-action is given by

$$
m \cdot(\gamma, \pi)=\alpha\left(\gamma^{-1}\right) m \pi, \quad(\gamma, \pi) \in G, \quad m \in M
$$

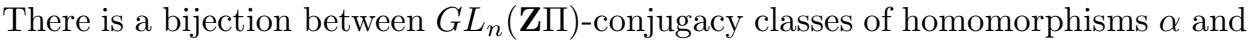
isomorphism classes of $\mathbf{Z} G$-lattices $M$ which satisfy

(a) $G / N$ acts trivially on the $N$-fixed points $M^{N}$, and

(b) $\operatorname{res}_{N} M$ is a free $\mathbf{Z} N$-module.

(See $[\mathrm{S}, \S 38.6]$ for details.)

Received by the editors November 1, 1997.

1991 Mathematics Subject Classification. Primary 20C10, 20C05; Secondary 16S34, $20 \mathrm{H} 25$.

Key words and phrases. Finite group, module, character.

This research was supported by grants from the Natural Sciences and Engineering Research Council of Canada. 
Definition. Let $\mathcal{D}(\Gamma, \Pi)$ ( $\mathcal{D}$ for "double-action") be the set of characters of $\mathbf{Z} G$ modules $M$ which satisfy (a) and (b).

If $\sigma: \Gamma \rightarrow \Pi$ is a homomorphism, it is easy to show (Lemma 2.1 below) that the double action module $M(\sigma)$ is isomorphic to the permutation module ind ${ }_{[\sigma]}^{G} \mathbf{Z}$, where $[\sigma]$ denotes the subgroup $\{(\gamma, \sigma(\gamma)): \gamma \in \Gamma\}$ of $G$. Define

$$
\begin{aligned}
R(G) & =\text { the virtual complex characters of } G, \\
R^{+}(G) & =\text { the proper characters of } G, \\
\mathcal{P}(\Gamma, \Pi) & =\mathbf{Z} \text {-span of }\left\{\operatorname{ind}_{[\sigma]}^{G} 1: \sigma \in \operatorname{hom}(\Gamma, \Pi)\right\} \subset R(G), \\
\mathcal{P}^{+}(\Gamma, \Pi) & =\mathbf{Z}_{\geq 0} \text {-span of }\left\{\operatorname{ind}_{[\sigma]}^{G} 1: \sigma \in \operatorname{hom}(\Gamma, \Pi)\right\} \subset R^{+}(G) .
\end{aligned}
$$

Here $\mathcal{P}$ is for "permutation". Then $\mathcal{P}^{+}$is contained in $\mathcal{D}$; it follows from [WAn] that if $\Pi$ is a $p$-group, then $\mathcal{D}=\mathcal{P}^{+}$. Indeed, it turns out (Proposition 2.3 below) that there is a positive answer to Problem 0 for finite groups $\Gamma, \Pi$ if and only if $\mathcal{D}=\mathcal{P}^{+}$. Note that $\mathcal{D}$ is closed under addition, and is a sub-semigroup of $R^{+}(G)$. Problem 0 can be reformulated as

Problem 1. Is $\mathcal{D}=\mathcal{P}^{+}$?

In $\S 6$ we give some conditions under which Problem 1 has a positive solution. In $\S 5$ we give examples of groups $\Gamma$ and $\Pi$ for which $\mathcal{D} \neq \mathcal{P}^{+}$. A counterexample to Problem 1 for $n=1$ has been constructed by Roggenkamp and Scott [RS] (see [K]); there are also such examples in $[\mathrm{B}]$, but with no general principles of construction for them. We will show that for $n>1$ counterexamples are so plentiful that our focus shifts to describing all of them. The feasibility of doing so is addressed by

Problem 2. Is the semigroup $\mathcal{D}$ finitely generated?

We will show that this is indeed true if $\Gamma$ and $\Pi$ are nilpotent; this is in $\S 7$. If we know that $\mathcal{D}$ is finitely generated, we are also interested in explicitly finding a generating set.

Analysis of $\mathcal{D}(\Gamma, \Pi)$ is complicated by questions about locally free class groups, so we are led to replace (b) in the definition of $\mathcal{D}$ by

$\left(\mathrm{b}^{\prime}\right) \operatorname{res}_{N} M$ is a locally free $\mathbf{Z} N$-module.

Definition. Let $\mathcal{D}^{\prime}(\Gamma, \Pi)$ be the set of characters of $\mathbf{Z} G$-modules $M$ which satisfy (a) and $\left(b^{\prime}\right)$.

The semigroup $\mathcal{D}^{\prime}$ is a good approximation to $\mathcal{D}$, in the sense that $r \mathcal{D}^{\prime} \subseteq \mathcal{D} \subseteq \mathcal{D}^{\prime}$ for some positive integer $r$ (Lemma 2.5 below); in other words, if $M$ is a $\mathbf{Z} \bar{G}$-module satisfying (a) and $\left(\mathrm{b}^{\prime}\right)$, then the direct sum $M^{r}$ of $r$ copies of $M$ satisfies (a) and (b). We get first approximations to results about $\mathcal{D}$ by considering $\mathcal{D}^{\prime}$ instead. All known cases of the equality $\mathcal{D}=\mathcal{P}^{+}$actually have $\mathcal{D}^{\prime}=\mathcal{P}^{+}$.

One of our main results, Theorem 3.3 below, is a complete character-theoretic description of $\mathcal{D}^{\prime}$ in the case that $\Pi$ is nilpotent. To explain this we introduce some notation. For a finite nilpotent group $N$, let $N_{p}$ be its Sylow $p$-subgroup and $N_{p^{\prime}}$ its Sylow $p$-complement. Then we have $R(G)=R\left(G_{p^{\prime}}\right) \otimes R\left(G_{p}\right)$, and we use this to define

$$
\begin{aligned}
\mathcal{Q}_{p}(\Gamma, \Pi) & =R\left(G_{p^{\prime}}\right) \otimes \mathcal{P}\left(\Gamma_{p}, \Pi_{p}\right) \\
\mathcal{Q}_{p}^{+}(\Gamma, \Pi) & =\left\{\sum \eta \otimes \lambda: \eta \in R^{+}\left(G_{p^{\prime}}\right), \lambda \in \mathcal{P}^{+}\left(\Gamma_{p}, \Pi_{p}\right)\right\}
\end{aligned}
$$


We show in Theorem 3.3 below that if $\Pi$ is nilpotent, then

$$
\mathcal{D}^{\prime}(\Gamma, \Pi)=\bigcap_{p} \mathcal{Q}_{p}^{+}(\Gamma, \Pi) .
$$

Theorem 3.3 is crucial to most of the results in the rest of the paper.

We are also interested in knowing if Problem 1 has a "virtual" answer, that is, is the $\mathbf{Z}$-span of $\mathcal{D}$ equal to $\mathcal{P}$ ? This is discussed in $\S 4$.

Although the above definitions and problems are sensible for arbitrary finite groups $\Gamma$ and $\Pi$, we shall need to assume that these groups are nilpotent for most of our results. This is because we rely on the local results of [WAn], which at this point have no analogues in the general case. From $\S 3$ through to the end of the paper, we will assume that $\Gamma$ and $\Pi$ are nilpotent.

\section{Preliminaries}

As in the introduction, let $G=\Gamma \times \Pi$ and $N=1 \times \Pi$. In this section, $\Pi$ and $\Gamma$ can be arbitrary finite groups.

Lemma 2.1. For $\sigma \in \operatorname{hom}(\Gamma, \Pi)$, the double action module $M(\sigma)$ is isomorphic to $\operatorname{ind}_{[\sigma]}^{G} \mathbf{Z}$ as $\mathbf{Z} G$-modules.

Proof. By definition, $M(\sigma)$ has $\mathbf{Z}$-basis $\Pi$. Consider elements of $\operatorname{ind}_{[\sigma]}^{G} \mathbf{Z}$ as linear combinations of the cosets of $[\sigma]$ in $G$; use $1 \times \Pi$ as coset representatives. Define

$$
f: M(\sigma) \rightarrow \operatorname{ind}_{[\sigma]}^{G} \mathbf{Z}, \quad f(\pi)=[\sigma](1, \pi), \quad \pi \in \Pi .
$$

To check that $f$ is a $\mathbf{Z} G$-homomorphism, for $\left(\gamma, \pi^{\prime}\right) \in G$,

$$
\begin{aligned}
f\left(\pi \cdot\left(\gamma, \pi^{\prime}\right)\right) & =f\left(\sigma\left(\gamma^{-1}\right) \pi \pi^{\prime}\right)=[\sigma]\left(1, \sigma\left(\gamma^{-1}\right) \pi \pi^{\prime}\right) \\
& \left.=[\sigma](\gamma, \sigma(\gamma))\left(1, \sigma\left(\gamma^{-1}\right) \pi \pi^{\prime}\right)\right)=[\sigma]\left(\gamma, \pi \pi^{\prime}\right)=f(\pi) \cdot\left(\gamma, \pi^{\prime}\right) .
\end{aligned}
$$

This proves the lemma.

Corollary 2.2. $\mathcal{P}^{+} \subseteq \mathcal{D}$.

Proposition 2.3. For a homomorphism $\alpha: \Gamma \rightarrow S G L_{n}(\mathbf{Z} \Pi)$, the character $\chi$ of the double action module $M(\alpha)$ is in $\mathcal{P}^{+}(\Gamma, \Pi)$ if and only if there exist group homomorphisms $\sigma_{i} \in \operatorname{hom}(\Gamma, \Pi), 1 \leq i \leq n$, and an element $u \in G L_{n}(\mathbf{Q} \Pi)$ such that $u \alpha(\gamma) u^{-1}=\operatorname{diag}\left(\sigma_{1}(\gamma), \ldots, \sigma_{n}(\gamma)\right)$ for all $\gamma \in \Gamma$.

Proof. Suppose that $\chi$ is in $\mathcal{P}^{+}(\Gamma, \Pi)$. By Lemma 2.1 there are homomorphisms $\sigma_{i}: \Gamma \rightarrow \Pi, 1 \leq i \leq k$, such that there is a $\mathbf{Q} G$-isomorphism

$$
f: \mathbf{Q} \otimes M(\alpha) \rightarrow \mathbf{Q} \otimes\left(\bigoplus_{i=1}^{k} M\left(\sigma_{i}\right)\right) .
$$

Comparing dimensions over $\mathbf{Q}$ gives $k=n$. Let $\left\{e_{j}: 1 \leq j \leq n\right\}$ be the standard basis of the $\mathbf{Q} \Pi$-column vectors $\mathbf{Q} \otimes M(\alpha)$, and write elements of $\mathbf{Q} \otimes M\left(\sigma_{i}\right)$ as $\langle x\rangle_{i}, x \in \mathbf{Q} \Pi$. Then $\mathbf{Q} \otimes\left(\bigoplus_{i=1}^{k} M\left(\sigma_{i}\right)\right)$ has $\mathbf{Q} N$-basis $\left\{\langle 1\rangle_{i}: 1 \leq i \leq n\right\}$ and, since $f$ is a $\mathbf{Q} N$-homomorphism, we have

$$
f\left(e_{j}\right)=\sum_{i}\left\langle u_{i j}\right\rangle_{i} \quad \text { where } u \in G L_{n}(\mathbf{Q} \Pi) .
$$


Act by $\left(\gamma^{-1}, 1\right)$, giving

$$
f\left(e_{j}\right)\left(\gamma^{-1}, 1\right)=\sum_{i}\left\langle u_{i j}\right\rangle_{i}\left(\gamma^{-1}, 1\right)=\sum_{i}\left\langle\sigma_{i}(\gamma) u_{i j}\right\rangle_{i} .
$$

Since $f\left(e_{j}\right)\left(\gamma^{-1}, 1\right)=f\left(e_{j}\left(\gamma^{-1}, 1\right)\right)$, this equals

$$
\begin{aligned}
f\left(\alpha(\gamma) e_{j}\right) & =f\left(\sum_{k} e_{k} \alpha(\gamma)_{k j}\right)=\sum_{k} f\left(e_{k}\right)\left(1, \alpha(\gamma)_{k j}\right) \\
& =\sum_{k, i}\left\langle u_{i k}\right\rangle_{i}\left(1, \alpha(\gamma)_{k j}\right)=\sum_{i}\left\langle\sum_{k} u_{i k} \alpha(\gamma)_{k j}\right\rangle_{i} .
\end{aligned}
$$

Therefore for all $i, j$ we have

$$
\sigma_{i}(\gamma) u_{i j}=\sum_{k} u_{i k} \alpha(\gamma)_{k j} \quad \text { so, as matrix equation, } \quad \operatorname{diag}\left(\sigma_{i}(\gamma)\right) u=u \alpha(\gamma) .
$$

This implies that $u \alpha(\gamma) u^{-1}=\operatorname{diag}\left(\sigma_{i}(\gamma)\right)$.

For the converse, given $u \in G L_{n}(\mathbf{Q} \Pi)$ such that $u \alpha(\gamma) u^{-1}=\operatorname{diag}\left(\sigma_{i}(\gamma)\right)$, define

$$
f: \mathbf{Q} \otimes M(\alpha) \rightarrow \mathbf{Q} \otimes\left(\oplus_{i} M\left(\sigma_{i}\right)\right)
$$

by $f\left(e_{j}\right)=\sum_{i}\left\langle u_{i j}\right\rangle_{i}$. It follows as above that $f$ is a $\mathbf{Q} G$-isomorphism.

Lemma 2.4. Suppose that $\Pi^{\prime}$ is a subgroup of $\Pi$, and set $G^{\prime}=\Gamma \times \Pi^{\prime}, N^{\prime}=1 \times \Pi^{\prime}$. Then $\operatorname{ind}_{G^{\prime}}^{G} \mathcal{D}\left(\Gamma, \Pi^{\prime}\right) \subseteq \mathcal{D}(\Gamma, \Pi)$.

Proof. We have $G=N G^{\prime}$ and $N \cap G^{\prime}=N^{\prime}$. Let $\chi^{\prime} \in \mathcal{D}\left(\Gamma, \Pi^{\prime}\right)$ be the character of a $\mathbf{Z} G^{\prime}$-lattice $M^{\prime}$ which satisfies (a) and (b) for $G^{\prime}, N$ in the definition of $\mathcal{D}$ in $\S 1$. We must check that $M=\operatorname{ind}_{G^{\prime}}^{G} M^{\prime}$ satisfies (a) and (b) for $G, N$.

For (a) identify $G / N$ and $G^{\prime} / N^{\prime}$ with $\Gamma$; there are $\Gamma$-isomorphisms

$$
M^{N} \cong M \otimes_{\mathbf{Z} G} \mathbf{Z} \Gamma \cong M^{\prime} \otimes_{\mathbf{Z} G^{\prime}} \mathbf{Z} G \otimes_{\mathbf{Z} G} \mathbf{Z} \Gamma \cong M^{\prime N^{\prime}},
$$

so $\Gamma$ acts trivially.

For (b), use Mackey decomposition to get

$$
\operatorname{res}_{N}^{G} M \cong \operatorname{res}_{N}^{G} \operatorname{ind}_{G^{\prime}}^{G} M^{\prime} \cong \operatorname{ind}_{N^{\prime}}^{N} \operatorname{res}_{N^{\prime}}^{G^{\prime}} M^{\prime},
$$

because $G=N G^{\prime}$ and $N \cap G^{\prime}=N^{\prime}$.

Lemma 2.5. $r \mathcal{D}^{\prime} \subseteq \mathcal{D} \subseteq \mathcal{D}^{\prime}$ for some positive integer $r$.

Proof. By definition, we have $\mathcal{D} \subseteq \mathcal{D}^{\prime}$. We will find a positive integer $r$ so that for any locally free $\mathbf{Z} N$-lattice $X, X^{r}$ is a free $\mathbf{Z} N$-lattice. Let $e$ be the exponent of the locally free class group of $\mathbf{Z} N$, as defined in [CR, 49.10]. Then $Y=X^{e}$ is stably free. From the Bass Cancellation Theorem [CR, 41.20] $Y \oplus Y$ is free, so $X^{2 e}$ is free, and the lemma is proved, with $r=2 e$.

To proceed further, we will apply the $p$-group results of [WAn]. In order to do this for all primes dividing $|\Pi|$, we will assume that $\Pi$ is nilpotent. We next show that under this assumption, $\alpha(\Gamma)$ is also nilpotent.

Lemma 2.6. Suppose that $\Pi$ is nilpotent; let $\phi_{p}$ denote the natural map

$$
\phi_{p}: G L_{n}(\mathbf{Z} \Pi) \rightarrow G L_{n}\left(\mathbf{Z}\left[\Pi / \Pi_{p}\right]\right) .
$$

Let $H$ be a finite subgroup of $S G L_{n}(\mathbf{Z \Pi )}$. Then the following hold:

1. $\operatorname{ker} \phi_{p} \cap H$ is a p-group. 
2. If $x \in S G L_{n}(\mathbf{Z} \Pi)$ has prime order $r$, then $r$ divides $|\Pi|$.

3. $\operatorname{ker} \phi_{p} \cap H$ is a normal Sylow p-subgroup of $H$.

Proof. 1. Suppose that $x \in \operatorname{ker} \phi_{p} \cap H$. Then $x=1+\delta$, where all the entries of $\delta$ are in $\mathbf{Z} \Pi \Delta\left(\Pi_{p}\right)$, where $\Delta\left(\Pi_{p}\right)$ is the augmentation ideal of $\mathbf{Z} \Pi_{p}$. Since $\mathbf{Z} \Pi \Delta\left(\Pi_{p}\right)$ is a nilpotent ideal $\bmod p$, it follows that for a suitable positive integer $m$ we have $x^{p^{m}}=1+p y$, for some element $y$ of the matrix ring $M_{n}(\mathbf{Z} \Pi)$. Then, raising to $p$-powers, we get

$$
x^{p^{m+i}}=1+p^{i} y_{i}, \quad y_{i} \in M_{n}(\mathbf{Z} \Pi)
$$

Now $x$ has finite order, since it is in the finite group $H$; so there are only finitely many possible values of $x^{p^{m+i}}$ as $i$ varies. Then there is a subsequence of integers $i$ for which $x^{p^{m+i}}$ is constant, say $z$. We see from the last equation that $z-1$ has coefficients divisible by arbitrarily high powers of $p$. This forces $z=1$, and $x$ has p-power order.

2. Use induction on $\Pi$. If $|\Pi|=1$, then $\left|S G L_{n}(\mathbf{Z} \Pi)\right|=1$. Suppose that $|\Pi|>1$. Let $p$ be a prime dividing $|\Pi|$. If $x \in \operatorname{ker} \phi_{p}$, then $x$ has order $p$ by 1 , and $r=p$. If $x \notin \operatorname{ker} \phi_{p}$, then $\phi_{p}(x) \in S G L_{n}\left(\mathbf{Z}\left[\Pi / \Pi_{p}\right]\right)$ has order $r$ dividing $\left|\Pi / \Pi_{p}\right|$, by induction.

3. Let $\Phi_{p}=\operatorname{ker} \phi_{p} \cap H$. From $1, \Phi_{p}$ is a $p$-group. Suppose that $y$ is an element of $p$-power order in $H$. From 2, $S G L_{n}\left(\mathbf{Z}\left[\Pi / \Pi_{p}\right]\right)$ has no element of order $p$; therefore $y \in \operatorname{ker} \phi_{p}$. So $\Phi_{p}$ contains all Sylow $p$-subgroups of $H$. This completes the proof.

Corollary 2.7. If $\Pi$ is nilpotent then $\mathcal{D}(\Gamma, \Pi)=\mathcal{D}\left(\Gamma_{\text {nil }}, \Pi\right)$, where $\Gamma_{\text {nil }}$ is the largest nilpotent quotient of $\Gamma$. The same holds for $\mathcal{D}^{\prime}$.

Proof. If $\chi \in \mathcal{D}(\Gamma, \Pi)$ is the character of $M(\alpha)$ then $\alpha(\Gamma)$ is nilpotent by Lemma 2.6 , since each Sylow subgroup is normal. Thus $\chi \in \mathcal{D}\left(\Gamma_{\text {nil }}, \Pi\right)$. The same assertion for $\mathcal{D}^{\prime}$ follows from Lemma 2.5 .

\section{Character-theoretic Description of $\mathcal{D}^{\prime}$}

For the rest of the paper, we assume that $\Pi$ is nilpotent; then by Corollary 2.7, it is no loss of generality to assume that $\Gamma$ is nilpotent. Thus we shall always assume that $\Gamma$ is nilpotent.

In this section we give a character-theoretic description of $\mathcal{D}^{\prime}(\Gamma, \Pi)$.

Lemma 3.1. Let $\chi \in R(G)=R\left(G_{p^{\prime}}\right) \otimes R\left(G_{p}\right)$ be written uniquely in the form

$$
\chi=\sum_{\eta \in \operatorname{irr}\left(G_{p^{\prime}}\right)} \eta \otimes \lambda_{\eta}, \quad \lambda_{\eta} \in R\left(G_{p}\right) .
$$

Then $\chi \in \mathcal{Q}_{p}^{+}(\Gamma, \Pi)$ if and only if $\lambda_{\eta} \in \mathcal{P}^{+}\left(\Gamma_{p}, \Pi_{p}\right)$ for all $\lambda$.

Proof. Since $R\left(G_{p^{\prime}}\right)$ has $\mathbf{Z}$-basis the irreducible complex characters $\operatorname{irr}\left(G_{p^{\prime}}\right)$, and $R^{+}\left(G_{p^{\prime}}\right)$ is the non-negative linear combinations of $\operatorname{irr}\left(G_{p^{\prime}}\right)$, the result follows.

Lemma 3.2. $\bigcap_{p} \mathcal{Q}_{p} \subseteq \mathbf{Q} \otimes \mathcal{P}$.

Proof. Take $\chi \in \bigcap_{p} \mathcal{Q}_{p}$. Let $B_{p}$ be a maximal linearly independent subset of $\left\{\operatorname{ind}_{\left[\sigma_{p}\right]}^{G_{p}} 1: \sigma_{p} \in \operatorname{hom}\left(\Gamma_{p}, \Pi_{p}\right)\right\}$; extend this to a basis $\widehat{B}_{p}$ of $\mathbf{Q} \otimes R\left(G_{p}\right)$. Then $\bigotimes_{p} \widehat{B}_{p}$ is a $\mathbf{Q}$-basis of $\bigotimes_{p}\left(\mathbf{Q} \otimes R\left(G_{p}\right)\right)=\mathbf{Q} \otimes R(G)$, so we can write $\chi$ uniquely as a $\mathbf{Q}$-linear combination in this basis. For a fixed $p, \chi$ is a linear combination of elements in 
$\left(\bigotimes_{l \neq p} \widehat{B}_{l}\right) \otimes B_{p}$. Varying over $p$ shows that $\chi$ is a $\mathbf{Q}$-linear combination of elements of $\otimes B_{p}$. A collection $\left\{\sigma_{p}: \Gamma_{p} \rightarrow \Pi_{p}\right\}$, one for each prime, corresponds to a single homomorphism $\sigma: \Gamma \rightarrow \Pi$, whose restriction to $\Gamma_{p}$ is $\sigma_{p}$. So $\chi \in \mathbf{Q} \otimes \mathcal{P}(\Gamma, \Pi)$.

Theorem 3.3. Suppose that $\Pi$ is nilpotent. Then $\mathcal{D}^{\prime}=\bigcap_{p} \mathcal{Q}_{p}^{+}$.

Proof. We first show that $\mathcal{D}^{\prime} \subseteq \bigcap_{p} \mathcal{Q}_{p}^{+}$. Suppose that $\chi \in \mathcal{D}^{\prime}$, and let $M$ be a $\mathbf{Z} G$-lattice affording $\chi$. Fix a prime $p$ dividing $|\Pi|$. We want to apply Theorem 2 of [WAn] to the $\mathbf{Z}_{p} G_{p}$-module $M_{p}=\operatorname{res}_{G_{p}}\left(\mathbf{Z}_{p} \otimes_{\mathbf{Z}} M\right)$, relative to the normal subgroup $N_{p}=1 \times \Pi_{p}$ of $G_{p}$. We need to verify that

(a) the $N_{p}$-fixed points $M_{p}^{N_{p}}$ have trivial $G_{p} / N_{p}$-action, and

(b) res ${ }_{N_{p}} M_{p}$ is a free $\mathbf{Z}_{p} N_{p}$-module.

Now (b) holds because res ${ }_{N} M$ is locally free. To prove (a), it is no loss to replace $M$ by a direct sum of copies of $M$, and by Lemma 2.5 , we may assume that $\operatorname{res}_{N} M$ is free. Then $M \cong M(\alpha)$ for some $\alpha: \Gamma \rightarrow G L_{n}(\mathbf{Z} \Pi)$; hence $M^{N_{p}} \cong M\left(\phi_{p} \alpha\right)$, where $\phi_{p}$ is as in Lemma 2.6. Now $\Gamma_{p}$ is in the kernel of $\phi_{p} \alpha$ by Lemma 2.6, so $G_{p} / N_{p}$ acts trivially on $M^{N_{p}}$.

From Theorem 2 of [WAn], $M_{p}$ is a permutation $\mathbf{Z}_{p} G_{p}$-lattice; moreover, the permuted basis is a disjoint union of orbits whose point stabilizers are of the form $\left[\sigma_{p}\right]=\left\{\left(\gamma, \sigma_{p}(\gamma)\right): \gamma \in \Gamma_{p}\right\}$, where $\sigma_{p} \in \operatorname{hom}\left(\Gamma_{p}, \Pi_{p}\right)$. Since $\mathbf{Z}_{p} \otimes M$ is a summand of ind $G_{G_{p}}^{G} M_{p}$, then $\mathbf{Z}_{p} \otimes M$ is a summand of a permutation $\mathbf{Z}_{p} G$-lattice.

Let $L$ be an indecomposable summand of $\mathbf{Z}_{p} \otimes M$. Denote its vertex by $1 \times D \subseteq$ $G_{p^{\prime}} \times G_{p}$; then $L$ is isomorphic to a summand of ind ${ }_{1 \times D}^{G_{p^{\prime}} \times G_{p}} \mathbf{Z}_{p} \cong \mathbf{Z}_{p} G_{p^{\prime}} \otimes$ ind $_{D}^{G_{p}} \mathbf{Z}_{p}$. Write $\mathbf{Z}_{p} G_{p^{\prime}}=\bigoplus X_{i}$ as a direct sum of (projective) indecomposables. Then $L$ is a summand of $X_{i} \otimes \operatorname{ind}_{D}^{G_{p}} \mathbf{Z}_{p}$ for some $i$. We claim that $Y=X_{i} \otimes \operatorname{ind}_{D}^{G_{p}} \mathbf{Z}_{p}$ is indecomposable. The $G_{p}$-fixed points of $Y$ are $X_{i} \otimes \mathbf{Z}_{p} \cong X_{i}$, which is irreducible $\bmod p$ as an $\mathbf{F}_{p} G_{p^{\prime}}$-module, since $p$ does not divide $\left|G_{p^{\prime}}\right|$. If $Y$ were decomposable, it would be decomposable $\bmod p$, say as $Z_{1} \oplus Z_{2}$, where $Z_{1}, Z_{2}$ are nonzero $\mathbf{F}_{p} G$ modules. The $G_{p}$-fixed points of each of $Z_{1}, Z_{2}$ are non-zero, since $G_{p}$ is a $p$-group. This contradicts the irreduciblity of $(Y / p Y)^{G_{p}}$ as $\mathbf{F}_{p} G_{p^{\prime}}$-module. So $Y$ is indeed indecomposable, and therefore $L$, which is a summand of $Y$, is $Y$ itself. Thus $\mathbf{Z}_{p} \otimes M$ is isomorphic to a sum of modules of the form $X \otimes$ ind $_{D}^{G_{p}} \mathbf{Z}_{p}$, where $X$ is a $\mathbf{Z}_{p} G_{p^{\prime}}$-module. Since each $D$ is of the form $\left[\sigma_{p}\right]$, it follows that

$$
\mathbf{z}_{p} \otimes M \cong \bigoplus_{\sigma_{p} \in \operatorname{hom}\left(\Gamma_{p}, \Pi_{p}\right)} X_{\sigma_{p}} \otimes \operatorname{ind}_{\left[\sigma_{p}\right]}^{G_{p}} \mathbf{z}_{p},
$$

where each $X_{\sigma_{p}}$ is a $\mathbf{Z}_{p} G_{p^{\prime}}$-module. Let $\xi_{\sigma_{p}}$ be the character of $X_{\sigma_{p}}$. Then $\chi$ has the form

$$
\chi(g)=\sum_{\sigma_{p}} \xi_{\sigma_{p}}\left(g_{p^{\prime}}\right) \operatorname{ind}_{\left[\sigma_{p}\right]}^{G_{p}} 1\left(g_{p}\right) .
$$

In other words, writing $R(G)=R\left(G_{p^{\prime}}\right) \otimes R\left(G_{p}\right)$, we have

$$
\chi=\sum_{\sigma_{p} \in \Sigma_{p}} \xi_{\sigma_{p}} \otimes \operatorname{ind}_{\left[\sigma_{p}\right]}^{G_{p}} 1, \quad \xi_{\sigma_{p}} \in R^{+}\left(G_{p^{\prime}}\right) .
$$

Write $\xi_{\sigma_{p}}$ as a sum of irreducible characters of $G_{p^{\prime}}$ :

$$
\xi_{\sigma_{p}}=\sum_{\eta \in \operatorname{irr}\left(G_{p^{\prime}}\right)} b\left(\sigma_{p}, \eta\right) \eta, \quad \text { with unique integers } b\left(\sigma_{p}, \eta\right) \geq 0 .
$$


Changing the order of summation, we have $\chi=\sum_{\eta} \eta \otimes \lambda_{\eta}$, where

$$
\lambda_{\eta}=\sum_{\sigma_{p}} b\left(\sigma_{p}, \eta\right) \operatorname{ind}_{\left[\sigma_{p}\right]}^{G_{p}} 1 \in \mathcal{P}^{+}\left(\Gamma_{p}, \Pi_{p}\right) .
$$

It follows from Lemma 3.1 that $\chi \in \bigcap_{p} \mathcal{Q}_{p}^{+}$.

Now suppose that $\chi \in \bigcap_{p} \mathcal{Q}_{p}^{+}$; we will show that $\chi \in \mathcal{D}^{\prime}$. From Lemma 3.2, $\chi \in \mathbf{Q} \otimes \mathcal{P}$; since characters in $\mathcal{P}$ are characters of permutation modules, we see that $\chi$ is rational valued.

Let $p$ be a prime dividing $|G|$. Since $\chi$ is in $\mathcal{Q}_{p}^{+}$, write $\chi$ as

$$
\chi=\sum_{\eta \in \operatorname{irr}\left(G_{p^{\prime}}\right)} \eta \otimes \lambda_{\eta}, \quad \lambda_{\eta} \in \mathcal{P}^{+}\left(\Gamma_{p}, \Pi_{p}\right) .
$$

Let $\zeta$ be a primitive $|G|$-th root of unity; let $\mathcal{G}$ denote the Galois group of $\mathbf{Q}(\zeta)$ over $\mathbf{Q}$, and let $\mathcal{G}_{p}$ denote the Galois group of $\mathbf{Q}_{p}(\zeta)$ over $\mathbf{Q}_{p}$, where $\mathbf{Q}_{p}$ is the $p$-adic rationals. Since $\chi$ and $\lambda_{\eta}$ are rational valued, we have

$$
\chi=\chi^{\omega}=\sum_{\eta} \eta^{\omega} \otimes \lambda_{\eta}, \quad \omega \in \mathcal{G}_{p} .
$$

By uniqueness of the representation $\chi=\sum_{\eta} \eta \otimes \lambda_{\eta}$, it follows that $\lambda_{\eta}=\lambda_{\eta^{\omega}}$,

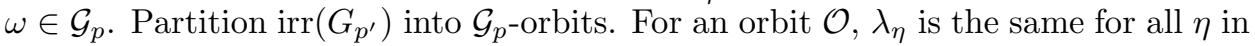
$\mathcal{O}$; call this common value $\lambda_{\mathcal{O}}$. Let $\tau_{\mathcal{O}}$ denote $\sum_{\eta \in \mathcal{O}} \eta$, which takes values in $\mathbf{Q}_{p}$. Then

$$
\chi=\sum_{\mathcal{O}} \sum_{\eta \in \mathcal{O}} \eta \otimes \lambda_{\eta}=\sum_{\mathcal{O}} \sum_{\eta \in \mathcal{O}} \eta \otimes \lambda_{\mathcal{O}}=\sum_{\mathcal{O}} \tau_{\mathcal{O}} \otimes \lambda_{\mathcal{O}} .
$$

We have each $\lambda_{\mathcal{O}} \in \mathcal{P}^{+}\left(\Gamma_{p}, \Pi_{p}\right)$, so we may choose non-negative integers $b\left(\sigma_{p}, \mathcal{O}\right)$ such that $\lambda_{\mathcal{O}}=\sum_{\sigma_{p}} b\left(\sigma_{p}, \mathcal{O}\right) \operatorname{ind}_{\left[\sigma_{p}\right]}^{G_{p}}$. Let

$$
\xi_{\sigma_{p}}=\sum_{\mathcal{O}} b\left(\sigma_{p}, \mathcal{O}\right) \tau_{\mathcal{O}}
$$

Then, changing the order of summation, we have

$$
\chi=\sum_{\mathcal{O}} \tau_{\mathcal{O}} \otimes \lambda_{\mathcal{O}}=\sum_{\mathcal{O}} \sum_{\sigma_{p}} \tau_{\mathcal{O}} \otimes b\left(\sigma_{p}, \mathcal{O}\right) \operatorname{ind}_{\left[\sigma_{p}\right]}^{G_{p}} 1=\sum_{\sigma_{p}} \xi_{\sigma_{p}} \otimes \operatorname{ind}_{\left[\sigma_{p}\right]}^{G_{p}} 1 .
$$

Now $\xi_{\sigma_{p}}$ is a $\mathbf{Z}_{\geq 0}$-linear combination of $\left\{\tau_{\mathcal{O}}\right\}$, and each $\tau_{\mathcal{O}}=\sum_{\eta \in \mathcal{O}} \eta$. Fix $\eta \in \operatorname{irr}\left(G_{p^{\prime}}\right)$ in an orbit $\mathcal{O}$. Since the order of $G_{p^{\prime}}$ is not divisible by $p$, the Schur index of $\eta$ over the field $\mathbf{Q}_{p}(\eta)$ is 1 , by [F, IV.9.5]. Let $K=\mathbf{Q}_{p}(\eta)$ and let $X$ be a $K G_{p^{\prime}}$-module affording $\eta$. By restriction of scalars, $X$ is a $\mathbf{Q}_{p} G_{p^{\prime}}$-module whose character is the sum of all the algebraic conjugates of $\eta$ over $\mathbf{Q}_{p}$, namely $\tau_{\mathcal{O}}$. Thus $\tau_{\mathcal{O}}$ is the character of a $\mathbf{Q}_{p} G_{p^{\prime}}$-module; hence so is $\xi_{\sigma_{p}}$. Choose a $\mathbf{Z}_{p} G_{p^{\prime}}$-lattice $L_{\sigma_{p}}$ in this module, so $\xi_{\sigma_{p}}$ is afforded by $L_{\sigma_{p}}$.

Next, define the $\mathbf{Z}_{p} G$-lattice $M(p)$ by

$$
M(p)=\bigoplus_{\sigma_{p}} L_{\sigma_{p}} \otimes_{\mathbf{z}_{p}} \operatorname{ind}_{\left[\sigma_{p}\right]}^{G_{p}} \mathbf{z}_{p} .
$$

We claim that

(a) the $N$-fixed points $M(p)^{N}$ have trivial $G / N$-action, and

(b) $\operatorname{res}_{N} M(p)$ is $\mathbf{Z}_{p} N$-free. 
To prove (a), note that $M(p)$ has character $\chi$ which is in $\bigcap_{p} \mathcal{Q}_{p}^{+}$; by Lemma 3.2, $\chi \in \mathbf{Q} \otimes \mathcal{P}$. Since the $N$-fixed points of ind ${ }_{[\sigma]}^{G} \mathbf{Z}$ have trivial $G / N$-action, (a) holds.

We now prove (b). Since $G_{p}=N_{p}\left[\sigma_{p}\right]$ and $N_{p} \cap\left[\sigma_{p}\right]=1$, it follows that $\operatorname{res}_{N_{p}}$ ind $_{\left[\sigma_{p}\right]}^{G_{p}} \mathbf{Z}_{p} \cong \mathbf{Z}_{p} N_{p}$ by Mackey decomposition. Since

$$
\operatorname{res}_{N} M(p) \cong \bigoplus_{\sigma_{p}}\left(\operatorname{res}_{N_{p^{\prime}}} L_{\sigma_{p}}\right) \otimes_{\mathbf{z}_{p}} \mathbf{z}_{p} N_{p} \cong \operatorname{res}_{N_{p^{\prime}}}\left(\bigoplus_{\sigma_{p}} L_{\sigma_{p}}\right) \otimes_{\mathbf{z}_{p}} \mathbf{z}_{p} N_{p}
$$

it suffices to show that res ${ }_{N_{p^{\prime}}}\left(\bigoplus_{\sigma_{p}} L_{\sigma_{p}}\right)$ is free over $\mathbf{Z}_{p} N_{p^{\prime}}$. Since $p$ does not divide $\left|N_{p^{\prime}}\right|$, we need only show that the character $\chi_{p}^{\prime}$ of $\bigoplus_{\sigma_{p}} L_{\sigma_{p}}$ has the property that res $_{N_{p^{\prime}}} \chi_{p}^{\prime}$ is a multiple of the character $\rho\left(N_{p^{\prime}}\right)$ of the regular representation of $N_{p^{\prime}}$. Since $\chi=\sum_{\sigma_{p}} \xi_{\sigma_{p}} \otimes$ ind $_{\left[\sigma_{p}\right]}^{G_{p}} 1$ relative to $R\left(G_{p^{\prime}}\right) \otimes R\left(G_{p}\right)$ and since the degree of each ind ${ }_{\left[\sigma_{p}\right]}^{G_{p}} 1$ is $\left|G_{p}:\left[\sigma_{p}\right]\right|=\left|\Pi_{p}\right|$, then $\operatorname{res}_{G_{p^{\prime}}} \chi=\left|\Pi_{p}\right| \chi_{p}^{\prime}$; hence res ${ }_{N_{p^{\prime}}} \chi_{p}^{\prime}$ is a Q-multiple of $\operatorname{res}_{N_{p^{\prime}}} \chi$. Let $y$ be a non-identity element of $N_{p^{\prime}}$. Then $y$ has the form $(1, \pi)$, so no conjugate of $y$ lies in $\left[\sigma_{p}\right]$. Since $\chi \in \mathbf{Q} \otimes \mathcal{P}$ by Lemma 3.2, $\chi$ and therefore $\chi_{p}^{\prime}$ both vanish on $y$. It follows that res $N_{p^{\prime}} \chi_{p}^{\prime}$ is a multiple of $\rho\left(N_{p^{\prime}}\right)$, as desired. This proves (b).

We next show that $\chi$ is afforded by a $\mathbf{Q} G$-module. As in equation (3.5), we have

$$
\chi=\sum_{\mathcal{O}^{\prime}} \tau_{\mathcal{O}^{\prime}} \otimes \lambda_{\mathcal{O}^{\prime}}
$$

where $\tau_{\mathcal{O}^{\prime}}$ is the orbit sum over an orbit of $\mathcal{G}$ acting on $\operatorname{irr}\left(G_{p^{\prime}}\right)$, and $\lambda_{\mathcal{O}^{\prime}}$ is the common value of $\lambda_{\eta}$ for all $\eta \in \mathcal{O}^{\prime}$. Let $R_{\mathbf{Q}}^{+}(G)$ be the characters afforded by $\mathrm{Q} G$-modules.

Since $\tau_{\mathcal{O}^{\prime}}$ is a rational valued character of $G_{p^{\prime}}$, then $\left|G_{p^{\prime}}\right| \tau_{\mathcal{O}^{\prime}} \in R_{\mathbf{Q}}^{+}\left(G_{p^{\prime}}\right)$; since $\lambda_{\mathcal{O}^{\prime}}$ is the character of a permutation module, then $\left|G_{p^{\prime}}\right| \chi \in R_{\mathbf{Q}}^{+}(G)$. Varying over $p$, since the greatest common divisor of the $\left|G_{p^{\prime}}\right|$ is 1 , it follows that $\chi \in R_{\mathbf{Q}}^{+}(G)$.

Let $V$ be a $\mathbf{Q} G$-module affording $\chi$. For each prime $p$ we have an isomorphism

$$
\phi_{p}: \mathbf{Q}_{p} \otimes_{\mathbf{Q}} V \rightarrow \mathbf{Q}_{p} \otimes_{\mathbf{z}_{p}} M(p) .
$$

For each $p$ let

$$
V(p)=\left\{v \in V: \phi_{p}(1 \otimes v) \in 1 \otimes M(p)\right\} .
$$

Then let $M=\bigcap_{p} V(p)$. From [R, 5.3] we see that $M$ is a $\mathbf{Z} G$-lattice such that $\mathbf{Z}_{p} \otimes M \cong M(p)$. Then $M$ affords $\chi$, the fixed points $M^{N}$ have trivial $G / N$-action, and $\operatorname{res}_{N}\left(\mathbf{Z}_{p} \otimes M\right) \cong \operatorname{res}_{N} M(p)$, so res ${ }_{N} M$ is locally free. Therefore $\chi$ is in $\mathcal{D}^{\prime}$, as desired. This completes the proof.

For later use, we record the following result, which is proved in the second paragraph of the proof of Theorem 3.3.

Proposition 3.4. If $M$ satisfies (a) and ( $\left.\mathrm{b}^{\prime}\right)$, then for each prime $p, \mathbf{Z}_{p} \otimes M$ is a summand of a permutation lattice for $\mathbf{Z}_{p} G$.

\section{The Lattice Spanned By $\mathcal{D}^{\prime}$}

In this section we show that the $\mathbf{Z}$-span $\mathbf{Z} \mathcal{D}^{\prime}$ of $\mathcal{D}^{\prime}$ is equal to $\bigcap_{p} \mathcal{Q}_{p}$. We also show that $\mathbf{Z} \mathcal{D}^{\prime}$ is equal to $\mathcal{P}$, if $\Gamma$ is cyclic. We do not know if this result holds for arbitrary $\Gamma$. 
Proposition 4.1. The $\mathbf{Z}$-span of $\mathcal{D}^{\prime}$ is equal to $\bigcap_{p} \mathcal{Q}_{p}$.

Proof. It follows from Theorem 3.3 that

$$
\mathbf{Z} \mathcal{D}^{\prime} \subseteq \bigcap_{p} \mathcal{Q}_{p}
$$

For the reverse inclusion, suppose that $\chi \in \bigcap_{p} \mathcal{Q}_{p}$. For a fixed $p$ dividing $|G|$, write

$$
\chi=\sum_{\eta \in \operatorname{irr}\left(G_{p^{\prime}}\right)} \eta \otimes \lambda_{\eta} \quad \text { for unique } \lambda_{\eta} \in \mathcal{P}\left(\Gamma_{p}, \Pi_{p}\right) .
$$

By Lemma 3.2, $\chi \in \mathbf{Q} \otimes \mathcal{P}$. For a given $\eta \in \operatorname{irr}\left(G_{p^{\prime}}\right)$, suppose that

$$
\left\langle\eta, \text { ind }_{\left[\sigma_{p^{\prime}}\right]}^{G_{p^{\prime}}} 1\right\rangle_{G_{p^{\prime}}}=0 \text { for all } \sigma_{p^{\prime}} \in \operatorname{hom}\left(\Gamma_{p^{\prime}}, \Pi_{p^{\prime}}\right) .
$$

Then $\eta$ is orthogonal to $\mathcal{P}\left(\Gamma_{p^{\prime}}, \Pi_{p^{\prime}}\right)$, and hence

$$
0=\left\langle\chi, \eta \otimes \lambda_{\eta}\right\rangle_{G}=\left\langle\eta \otimes \lambda_{\eta}, \eta \otimes \lambda_{\eta}\right\rangle_{G}=\langle\eta, \eta\rangle_{G_{p^{\prime}}}\left\langle\lambda_{\eta}, \lambda_{\eta}\right\rangle_{G_{p}}
$$

It follows that $\lambda_{\eta}=0$. Thus in equation (4.1), we need only sum over $\eta \in \operatorname{irr}\left(G_{p^{\prime}}\right)$ for which (4.2) does not hold. For such an $\eta$, if $\left\langle\eta \text {, ind }{ }_{\left[\sigma_{p^{\prime}}\right]}^{G_{p^{\prime}}} 1\right\rangle_{G_{p^{\prime}}} \neq 0$, decompose $\operatorname{ind}_{\left[\sigma_{p^{\prime}}\right]}^{G_{p^{\prime}}} 1$, giving

$$
-\eta=\sum \tilde{\eta}-\operatorname{ind}_{\left[\sigma_{p^{\prime}}\right]}^{G_{p^{\prime}}} 1
$$

for some $\tilde{\eta} \in \operatorname{irr}\left(G_{p^{\prime}}\right)$. Write $\lambda_{\eta}=\lambda_{\eta}^{\prime}-\lambda_{\eta}^{\prime \prime}$ with $\lambda_{\eta}^{\prime}, \lambda_{\eta}^{\prime \prime} \in \mathcal{P}^{+}\left(\Gamma_{p}, \Pi_{p}\right)$; we get

$$
\chi=\sum \eta \otimes \lambda_{\eta}^{\prime}+\sum(-\eta) \otimes \lambda_{\eta}^{\prime \prime} .
$$

In this equation, replace $-\eta$ using equation (4.3). We get $\chi=\chi_{p}^{\prime}-\xi_{p}$ with $\chi_{p}^{\prime} \in \mathcal{Q}_{p}^{+}$, $\xi_{p} \in \mathcal{P}^{+}$. Let $\xi=\sum_{p|| G \mid} \xi_{p} \in \mathcal{P}^{+}$; then $\chi+\xi=\chi_{p}^{\prime}+\sum_{l \neq p} \xi_{l}$, which is in $\mathcal{Q}_{p}^{+}$for all $p$. Thus

$$
\chi=(\chi+\xi)-\xi
$$

with $\chi+\xi \in \bigcap_{p} \mathcal{Q}_{p}^{+}$and $\xi \in \mathcal{P}^{+} \subseteq \bigcap_{p} \mathcal{Q}_{p}^{+}$. Since $\bigcap_{p} \mathcal{Q}_{p}^{+}=\mathcal{D}^{\prime}$ by Theorem 3.3, the result is proved.

We next show that $\mathbf{Z} \mathcal{D}^{\prime}=\mathcal{P}$ if $\Gamma$ is cyclic. Let $\Sigma$ be a complete set of homomorphisms from $\Gamma$ to $\Pi$ up to conjugacy in $\Pi$. Our proof that $\mathbf{Z} \mathcal{D}^{\prime}=\mathcal{P}$ for cyclic $\Gamma$ uses the next result, that $\left\{\operatorname{ind}_{[\sigma]}^{G} 1: \sigma \in \Sigma\right\}$ is linearly independent over $\mathbf{Q}$ if $\Gamma$ is cyclic. This lemma can fail if $\Gamma$ is not cyclic, but it is possible that $\mathbf{Z} \mathcal{D}^{\prime}=\mathcal{P}$ can be proved by some other method in the non-cyclic case.

Lemma 4.2. The set $\left\{\operatorname{ind}_{[\sigma]}^{G} 1: \sigma \in \Sigma\right\}$ is a basis of $\mathbf{Q} \otimes \mathcal{P}$ if $\Gamma$ is cyclic.

Proof. For $g=(\gamma, \pi) \in G$ and $\sigma \in \operatorname{hom}(\Gamma, \Pi)$, we have

$$
\operatorname{ind}_{[\sigma]}^{G} 1(g)= \begin{cases}\left|C_{\Pi}(\pi)\right|, & \sigma(\gamma) \sim \pi, \\ 0, & \text { else, }\end{cases}
$$

where $\sim$ denotes conjugacy in $\Pi$. If $\tau \in \operatorname{hom}(\Gamma, \Pi)$ then $\tau$ is conjugate to some $\sigma \in \Sigma$. From the formula for $\operatorname{ind}_{[\sigma]}^{G} 1(g)$ above, then $\operatorname{ind}_{[\tau]}^{G} 1=\operatorname{ind}_{[\sigma]}^{G} 1$, so $\mathbf{Q} \otimes \mathcal{P}$ is spanned by $\left\{\operatorname{ind}_{[\sigma]}^{G} 1: \sigma \in \Sigma\right\}$. 
Suppose that $\sum_{\sigma \in \Sigma} a_{\sigma} \operatorname{ind}_{[\sigma]}^{G} 1=0$ with $a_{\sigma} \in \mathbf{Q}$. Given $\tau \in \Sigma$, let $\gamma$ be a generator of $\Gamma$, and evaluate at $(\gamma, \tau(\gamma))$. We get

$$
\operatorname{ind}_{[\sigma]}^{G} 1(\gamma, \tau(\gamma))= \begin{cases}\left|C_{\Pi}(\tau(\gamma))\right|, & \sigma(\gamma) \sim \tau(\gamma), \\ 0, & \text { else. }\end{cases}
$$

If $\tau$ and $\sigma$ are distinct elements of $\Sigma$, then $\tau(\gamma)$ and $\sigma(\gamma)$ are not conjugate in $\Pi$, so $\operatorname{ind}_{[\sigma]}^{G} 1(\gamma, \tau(\gamma))=0$. It follows that $a_{\tau}=0$, for all $\tau$ in $\Sigma$, and the result is proved.

Proposition 4.3. The $\mathbf{Z}$-span of $\mathcal{D}^{\prime}$ is equal to $\mathcal{P}$ if $\Gamma$ is cyclic.

Proof. Since $\mathcal{P}^{+} \subseteq \mathcal{D}^{\prime}$ it suffices to show that $\mathcal{D}^{\prime} \subseteq \mathcal{P}$. Suppose that $\chi \in \mathcal{D}^{\prime}$. Then $\chi \in \mathbf{Q} \otimes \mathcal{P}$ from Theorem 3.3 and Lemma 3.2. Then $\chi=\sum_{\sigma \in \Sigma} a_{\sigma} \operatorname{ind}_{[\sigma]}^{G} 1$, where $a_{\sigma} \in \mathbf{Q}$, and by Lemma 4.2 , the $a_{\sigma} \in \mathbf{Q}$ are unique. We must show that each $a_{\sigma} \in \mathbf{Z}$.

Since $G$ is nilpotent, we pick $\Sigma$ by picking complete sets $\Sigma_{p} \subseteq \operatorname{hom}\left(\Gamma_{p}, \Pi_{p}\right)$ up to conjugacy in $\Pi_{p}$, and then letting $\Sigma$ be those homomorphisms whose restrictions to $\Gamma_{p}$ are in $\Sigma_{p}$.

Fix a prime $p$ dividing $|G|$. From Theorem 3.3, $\chi \in \mathcal{Q}_{p}(\Gamma, \Pi)=R\left(G_{p^{\prime}}\right) \otimes$ $\mathcal{P}\left(\Gamma_{p}, \Pi_{p}\right)$. For $\sigma \in \Sigma$, we have $\operatorname{ind}_{[\sigma]}^{G} 1=\operatorname{ind}_{\left[\sigma_{p^{\prime}}\right]}^{G G_{p^{\prime}}} 1 \otimes \operatorname{ind}_{\left[\sigma_{p}\right]}^{G_{p}} 1$. Then

$$
\chi=\sum_{\tau \in \Sigma_{p}}\left(\sum_{\substack{\sigma \in \Sigma \\ \sigma_{p}=\tau}} a_{\sigma} \operatorname{ind}_{\left[\sigma_{p^{\prime}}\right]}^{G_{p^{\prime}}} 1\right) \otimes \operatorname{ind}_{[\tau]}^{G_{p}} 1 .
$$

But $\left\{\operatorname{ind}_{[\tau]}^{G_{p}} 1\right\}$ is a $\mathbf{Z}$-basis of $\mathcal{P}\left(\Gamma_{p}, \Pi_{p}\right)$, from Lemma 4.2, so it follows that

$$
\phi_{\tau}=\sum_{\substack{\sigma \in \Sigma \\ \sigma_{p}=\tau}} a_{\sigma} \operatorname{ind}_{\left[\sigma_{p^{\prime}}\right]}^{G_{p^{\prime}}} 1 \in R\left(G_{p^{\prime}}\right)
$$

for all $\tau \in \Sigma_{p}$. Let $\gamma_{p^{\prime}}$ be a generator of $\Gamma_{p^{\prime}}$. Evaluate $\phi_{\tau}$ at $\left(\gamma_{p^{\prime}}, \sigma_{p^{\prime}}\left(\gamma_{p}^{\prime}\right)\right)$. For the unique $\sigma \in \Sigma$ whose restriction to $\Gamma_{p}$ is $\tau$ and whose restriction to $\Gamma_{p^{\prime}}$ is $\sigma_{p^{\prime}}$, we get, as in the proof of Lemma 4.1,

$$
\left|C_{\Pi_{p^{\prime}}}\left(\sigma_{p^{\prime}}\left(\gamma_{p^{\prime}}\right)\right)\right| a_{\sigma} \in \mathbf{Z}
$$

since the values of $\phi_{\tau}$ are algebraic integers in $\mathbf{Q}$. Thus

$$
\left|\Pi_{p^{\prime}}\right| a_{\sigma} \in \mathbf{Z}, \quad \text { for all } \sigma \in \Sigma .
$$

This holds for all $p$. Since the greatest common divisor of $\left|\Pi_{p^{\prime}}\right|$ is 1 , it follows that $a_{\sigma} \in \mathbf{Z}$ for all $\sigma \in \Sigma$, and the proof is complete.

Here is an example showing that Lemma 4.2 can fail if $\Gamma$ is not cyclic. Let $\Gamma$ and $\Pi$ each be the direct product $C_{p} \times C_{p}$ of cyclic groups of prime order. Consider the $p^{4} \times p^{4}$ matrix whose rows are indexed by $\Sigma=\operatorname{hom}(\Gamma, \Pi)$ and columns by $G=\Gamma \times \Pi$, with $(\sigma, g)$-entry given by $\operatorname{ind}_{[\sigma]}^{G} 1(g)$. Since $\operatorname{ind}_{[\sigma]}^{G} 1(1, x)=0$ if $x$ is a nontrivial element of $\Pi$, then there is a column of zeros, and so the rows are linearly dependent. 


\section{Examples of $\mathcal{D} \neq \mathcal{P}^{+}$}

In this section we produce examples of groups $\Gamma, \Pi$ and modules $M$ having character which is in $\mathcal{D}(\Gamma, \Pi)$ but not in $\mathcal{P}^{+}(\Gamma, \Pi)$.

Lemma 5.1. For distinct primes $p_{1}$ and $p_{2}$, let $\Gamma=C_{p_{1} p_{2}}$, the cyclic group of order $p_{1} p_{2}$, and let $\Pi=C_{p_{1} p_{2}} \times C_{p_{1} p_{2}}$. Then $\mathcal{D}(\Gamma, \Pi) \neq \mathcal{P}^{+}(\Gamma, \Pi)$.

Proof. As before, let $G=\Gamma \times \Pi, N=1 \times \Pi$. Choose sets $\Sigma_{1}, \Sigma_{2}$ of homomorphisms $\sigma: \Gamma \rightarrow \Pi$ whose images $\sigma(\Gamma)$ are precisely the subgroups of $\Pi$ of order $p_{1}$, respectively, $p_{2}$. Let $\Sigma=\Sigma_{1} \cup \Sigma_{2}$. Let $\tau: \Gamma \rightarrow \Pi$ be the trivial map. The module $M$ we will construct has character

$$
\chi=-\operatorname{ind}_{[\tau]}^{G} 1+\sum_{\sigma \in \Sigma} \operatorname{ind}_{[\sigma]}^{G} 1 .
$$

It will follow from our construction that $\chi \in \mathcal{D}(\Gamma, \Pi)$. Let $\gamma$ be a generator of $\Gamma$, and let $g=(\gamma, 1) \in G$. Then $(\gamma, 1) \in[\tau]$, so ind ${ }_{[\tau]}^{G} 1(g)=|\Pi|$, but $(\gamma, 1) \notin[\sigma]$ for all $\sigma \in \Sigma$, so $\operatorname{ind}_{[\sigma]}^{G} 1(g)=0$. Hence $\chi(g)<0$ and $\chi$ is not the character of a permutation module, so $\chi \notin \mathcal{P}^{+}(\Gamma, \Pi)$.

For $\sigma \in \Sigma, M(\sigma)$ is the corresponding double-action module. Define

$$
M(\Sigma)=\bigoplus_{\sigma \in \Sigma} M(\sigma) .
$$

For each $\sigma \in \Sigma$, let $s(\sigma)=\sum_{x \in \sigma(\Gamma)} x \in \mathbf{Z} \Pi$, and define the map

$$
f_{\sigma}: M(\sigma) \rightarrow M(\tau), \quad f_{\sigma}(m)=s(\sigma) m, \quad m \in M(\sigma) .
$$

Define $f: M(\Sigma) \rightarrow M(\tau)$ by $f=\sum_{\sigma} f_{\sigma}$. The key to the proof is the claim that $f$ is an epimorphism of $\mathbf{Z} G$-modules.

We now prove that the claim holds. Since $s(\sigma) \sigma\left(\gamma^{-1}\right)=s(\sigma)=\sigma\left(\gamma^{-1}\right) s(\sigma)$, then each $f_{\sigma}$ is a $\mathbf{Z} G$-homorphism, and so is $f$. To prove that $f$ is surjective, it suffices to find $v \in M(\Sigma)$ such that $f(v)=1 \in M(\tau)$. Pick two distinct elements $\phi_{i}, \psi_{i}$ from $\Sigma_{i}, i=1,2$. Find integers $n_{1}, n_{2}$ such that $n_{1} p_{1}+n_{2} p_{2}=1$. Define $v=\sum_{\sigma \in \Sigma} v_{\sigma}$ with $v_{\sigma} \in M(\sigma)$ given by

$$
v_{\sigma}= \begin{cases}n_{i} \cdot 1, & \sigma \in \Sigma_{i}, \quad \sigma \neq \phi_{i}, \\ n_{i}\left(1-s\left(\psi_{i}\right)\right), & \sigma=\phi_{i} .\end{cases}
$$

To compute $f(v)$,

$$
f(v)=n_{1} \sum_{\substack{\sigma \in \Sigma_{1} \\ \sigma \neq \phi_{1}}} s(\sigma)+n_{1} s\left(\phi_{1}\right)\left(1-s\left(\psi_{1}\right)\right)+n_{2} \sum_{\substack{\sigma \in \Sigma_{2} \\ \sigma \neq \phi_{2}}} s(\sigma)+n_{2} s\left(\phi_{2}\right)\left(1-s\left(\psi_{2}\right) .\right.
$$

Since

$$
s(\sigma) s(\tilde{\sigma})=\widehat{\Pi}_{p_{i}}, \quad \sigma, \tilde{\sigma} \in \Sigma_{i}, \sigma \neq \tilde{\sigma},
$$

we get

$$
f(v)=n_{1}\left(\sum_{\sigma \in \Sigma_{1}} s(\sigma)-\widehat{\Pi}_{p_{1}}\right)+n_{2}\left(\sum_{\sigma \in \Sigma_{2}} s(\sigma)-\widehat{\Pi}_{p_{2}}\right) .
$$


In the sum $\sum_{\sigma \in \Sigma_{i}} s(\sigma) \in \mathbf{Z}_{p_{i}}$, non-identity elements $y \in \Pi_{p_{i}}$ occur exactly once, whereas 1 occurs $p_{i}+1$ times and

$$
\sum_{\sigma \in \Sigma_{i}} s(\sigma)=p_{i} \cdot 1+\widehat{\Pi}_{p_{i}} .
$$

We obtain

$$
f(v)=n_{1} p_{1} \cdot 1+n_{2} p_{2} \cdot 1=1 .
$$

Therefore $f$ is indeed a $\mathbf{Z} G$-epimorphism.

Now define $M$ to be the kernel of $f: M(\Sigma) \rightarrow M(\tau)$, so we have the exact sequence

$$
0 \rightarrow M \rightarrow M(\Sigma) \stackrel{f}{\rightarrow} M(\tau) \rightarrow 0 .
$$

Since $\operatorname{res}_{N} M(\sigma)$ is free for all $\sigma$, this sequence splits when restricted to $N$; hence res $_{N} M$ is stably free. Since $\mathbf{Z} N$ satisfies the Eichler condition, it follows that $\operatorname{res}_{N} M$ is $\mathbf{Z} N$-free by Jacobinski's Cancellation Theorem [CR, 51.24]. (In fact, it can be shown directly that $\operatorname{res}_{N} M$ is $\mathbf{Z} N$-free by exhibiting a basis; this is done in a special case below.) Since $G / N$ acts trivially on $M(\Sigma)^{N}$, it does so on $M^{N}$. The character $\chi$ of $M$ is therefore in $\mathcal{D}$, and it follows from the exact sequence that $\chi=-\operatorname{ind}_{[\tau]}^{G} 1+\sum_{\sigma \in \Sigma} \operatorname{ind}_{[\sigma]}^{G} 1$. This completes the proof.

From Proposition 2.4, there exists $U \in S G L_{p_{1} p_{2}}(\mathbf{Z} \Pi)$ with $U^{p_{1} p_{2}}=1$ and $U$ not conjugate in $G L(\mathbf{Q} \Pi)$ to a diagonal matrix of group elements. We can exhibit such a matrix $U$ by computing the action of a generator of $\Gamma$ on an explicit $\mathbf{Z} N$-basis of the free module res ${ }_{N} M$. To simplify the exposition, we assume that

$$
p_{1}=2, \quad p_{2}=3 .
$$

Then pick

$$
n_{1}=-1, \quad n_{2}=1 .
$$

Write $\Gamma=\langle c\rangle$ of order $6, \Pi=\langle a\rangle \times\langle b\rangle$, where $a$ and $b$ each have order 6 . Let

$$
\begin{gathered}
\Sigma_{1}=\left\{\sigma_{1}, \sigma_{2}, \sigma_{3}\right\}, \quad \sigma_{1}(c)=a^{3}, \sigma_{2}(c)=b^{3}, \sigma_{3}(c)=a^{3} b^{3}, \\
\Sigma_{2}=\left\{\sigma_{4}, \sigma_{5}, \sigma_{6}, \sigma_{7}\right\}, \quad \sigma_{4}(c)=a^{2}, \sigma_{5}(c)=b^{2}, \sigma_{6}(c)=a^{2} b^{2}, \sigma_{7}(c)=a^{2} b^{4} .
\end{gathered}
$$

Pick

$$
\psi_{1}=\sigma_{1}, \quad \phi_{1}=\sigma_{3}, \quad \psi_{2}=\sigma_{4}, \quad \phi_{2}=\sigma_{7}
$$

Define

$$
s_{i}=s\left(\sigma_{i}\right), \quad c_{i}=\sigma_{i}(c) .
$$

Identify $M(\Sigma)$ with $\mathbf{Z} \Pi^{7}$ and $M(\tau)$ with $\mathbf{Z} \Pi$, Then our map $f$ takes $\mathbf{Z} \Pi^{7}$ to $\mathbf{Z} \Pi$, given by

$$
f\left(z_{1}, \ldots, z_{7}\right)^{T}=\sum_{i=1}^{7} s_{i} z_{i}
$$

The $G$-action on $\mathbf{Z} \Pi^{7}$ becomes

$$
\left(z_{1}, \ldots, z_{7}\right)^{T}(x, y)=\left(\sigma_{1}\left(x^{-1}\right) z_{1} y \cdots, \sigma_{7}\left(x^{-1}\right) z_{7} y\right)^{T} .
$$


(Transposes are used because $M(\Sigma)$ is a right $\mathbf{Z} \Pi$-module and endomorphisms are matrices over $\mathbf{Z} \Pi$ acting on the left.) In this notation the element $v$ in the proof of Lemma 5.1 is

$$
v=\left(-1,-1, s_{1}-1,1,1,1,1-s_{4}\right)^{T} .
$$

Let $\left\{e_{1}, e_{2}, \ldots, e_{7}\right\}$ be the standard basis of $\mathbf{Z} \Pi^{7}$. Extend the "unimodular column" $v$ to a $\mathbf{Z} \Pi$-basis $\left\{v, e_{2}, \ldots, e_{7}\right\}$ of $\mathbf{Z} \Pi^{7}$. Then do "elementary operations" by setting

$$
m_{i}=e_{i+1}-v f\left(e_{i+1}\right)=e_{i+1}-v s_{i+1}, \quad 1 \leq i \leq 6,
$$

and then $\left\{v, m_{1}, m_{2}, \ldots, m_{6}\right\}$ is a $\mathbf{Z} \Pi$-basis of $\mathbf{Z} \Pi^{7}$. The $m_{i}$ are in $M$ by construction, so $\left\{m_{1}, \ldots, m_{6}\right\}$ is a $\mathbf{Z} \Pi$-basis of $M$.

We want the action of a generator of $\Gamma$ on $M$ in this basis. The matrix of $\left(c^{-1}, 1\right)$ in the standard basis of $\mathbf{Z} \Pi^{7}$ is $D=\operatorname{diag}\left(c_{1}, \ldots, c_{7}\right)$. So we need only see the effect of two changes of basis. Set

$$
A=\left(\begin{array}{ccccccccc}
-1 & & & & & \\
-1 & 1 & & & & \\
s_{1}-1 & & 1 & & & \\
1 & & & 1 & & \\
1 & & & 1 & \\
1 & & & & 1 \\
1-s_{4} & & & & & 1
\end{array}\right), B=\left(\begin{array}{ccccccc}
1 & -s_{2} & -s_{3} & -s_{4} & -s_{5} & -s_{6} & -s_{7} \\
& 1 & & & & & \\
& & 1 & & & & \\
& & & 1 & & & \\
& & & & 1 & & \\
& & & & & 1 & \\
& & & & & & 1
\end{array}\right) \text {. }
$$

The action of $\left(c^{-1}, 1\right)$ in the basis $\left\{v, m_{1}, m_{2}, \ldots, m_{6}\right\}$ is given by the $\mathbf{Z} \Pi$-matrix $X=B^{-1} A^{-1} D A B$. Since $M$ is a $G$-submodule of $\mathbf{Z} \Pi^{7}$, this matrix has the form

$$
\left(\begin{array}{ccccccc}
1 & 0 & 0 & 0 & 0 & 0 & 0 \\
* & & & & & & \\
* & & & & & & \\
* & & & U & & & \\
* & & & & & & \\
* & & & & & & \\
* & & & & & &
\end{array}\right)
$$

for some $U \in G L_{6}(\mathbf{Z} \Pi)$, and it is this $U$ that we want. Using $c_{i} s_{i}=s_{i}$, and denoting $c_{i j}=c_{i}-c_{j}, s_{1}^{*}=s_{1}-1, s_{4}^{*}=s_{4}-1$, we get $U=$

$$
\left(\begin{array}{cccccc}
c_{2}+c_{21} s_{2} & c_{21} s_{3} & c_{21} s_{4} & c_{21} s_{5} & c_{21} s_{6} & c_{21} s_{7} \\
c_{13} s_{1}^{*} s_{2} & c_{3}+c_{13} s_{1}^{*} s_{3} & c_{13} s_{1}^{*} s_{4} & c_{13} s_{1}^{*} s_{5} & c_{13} s_{1}^{*} s_{6} & c_{13} s_{1}^{*} s_{7} \\
c_{14} s_{2} & c_{14} s_{3} & c_{4}+c_{14} s_{4} & c_{14} s_{5} & c_{14} s_{6} & c_{14} s_{7} \\
c_{15} s_{2} & c_{15} s_{3} & c_{15} s_{4} & c_{5}+c_{15} s_{5} & c_{15} s_{6} & c_{15} s_{7} \\
c_{16} s_{2} & c_{16} s_{3} & c_{16} s_{4} & c_{16} s_{5} & c_{6}+c_{16} s_{6} & c_{16} s_{7} \\
c_{71} s_{4}^{*} s_{2} & c_{71} s_{4}^{*} s_{3} & c_{71} s_{4}^{*} s_{4} & c_{71} s_{4}^{*} s_{5} & c_{71} s_{4}^{*} s_{6} & c_{7}+c_{71} s_{4}^{*} s_{7}
\end{array}\right) .
$$

Note that this matrix has trace $-1+\sum_{i=1}^{7} c_{i}$, so it is a counterexample to the strategy of [MRSW]. Actually every $\chi \in \mathcal{D}^{\prime}-\mathcal{P}^{+}$gives such a counterexample, by Lemma 1 of [WCr] generalized to matrices.

Lemma 5.2. Let $p$ be an odd prime, and let $\Gamma=C_{4} \times C_{p}$ and $\Pi=Q_{8} \times C_{p} \times C_{p}$, where $Q_{8}$ is the quaternion group of order 8 . Then $\mathcal{D}(\Gamma, \Pi) \neq \mathcal{P}^{+}(\Gamma, \Pi)$. 
Proof. Pick $\tau \in \operatorname{hom}(\Gamma, \Pi)$ whose image is one of the cyclic subgroups of order 4 of $Q_{8}$. Choose $\Sigma_{2}$ to consist of three homomorphisms $\Gamma \rightarrow \Pi$ whose images are the two other subgroups of order 4 in $Q_{8}$ as well as the subgroup of order 2. Choose $\Sigma_{p}$ to consist of $p+1$ elements of hom $(\Gamma, \Pi)$ so that im $\sigma_{p}$ are the nontrivial cyclic $p$-subgroups and so that $\sigma_{2}=\tau_{2}$. Set $\Sigma=\Sigma_{2} \cup \Sigma_{p}$, and

$$
\chi^{\prime}=-\operatorname{ind}_{[\tau]}^{G} 1+\sum_{\sigma \in \Sigma} \operatorname{ind}_{[\sigma]}^{G} 1 .
$$

Relative to $R(G)=R\left(G_{p}\right) \otimes R\left(G_{2}\right)$,

$$
\chi^{\prime}=\left(-\operatorname{ind}_{[1]}^{G_{p}} 1+\sum_{\sigma \in \Sigma_{p}} \operatorname{ind}_{\left[\sigma_{p}\right]}^{G_{p}} 1\right) \otimes \operatorname{ind}_{\left[\tau_{2}\right]}^{G_{2}} 1+\sum_{\sigma \in \Sigma_{2}} \operatorname{ind}_{[1]}^{G_{p}} 1 \otimes \operatorname{ind}_{\left[\sigma_{2}\right]}^{G_{2}} 1,
$$

where the expression in parentheses is in $R^{+}\left(G_{p}\right)$. So $\chi \in \mathcal{Q}_{2}^{+}$. Relative to $R(G)=$ $R\left(G_{2}\right) \otimes R\left(G_{p}\right)$ we have

$$
\chi^{\prime}=\left(-\operatorname{ind}_{\left[\tau_{2}\right]}^{G_{2}} 1+\sum_{\sigma \in \Sigma_{2}} \operatorname{ind}_{\left[\sigma_{2}\right]}^{G_{2}}\right) \otimes \operatorname{ind}_{[1]}^{G_{p}} 1+\sum_{\sigma \in \Sigma_{p}} \operatorname{ind}_{\left[\sigma_{p}\right]}^{G_{p}} 1 \otimes \operatorname{ind}_{\left[\tau_{2}\right]}^{G_{2}} 1,
$$

where the expression in parentheses is in $R^{+}\left(G_{2}\right)$. Therefore $\chi \in \mathcal{Q}_{p}^{+}$.

By Theorem 3.3, $\chi^{\prime} \in \mathcal{D}^{\prime}$; hence $\chi=r \chi^{\prime} \in \mathcal{D}$ by Lemma 2.5. We shall show that $\chi \notin \mathcal{P}^{+}$. By Lemma 4.2, it is enough to check that $\tau$ is not $\Pi$-conjugate to any $\sigma \in \Sigma$. But the image of $\tau$, which is normal in $\Pi$, is different from the images of all $\sigma \in \Sigma$. This completes the proof.

\section{On Problem 1}

In this section we prove that $\mathcal{D}^{\prime}=\mathcal{P}^{+}$if $\Pi$ is nilpotent and $\Gamma$ has prime-prower order. Since $\mathcal{P}^{+} \subseteq \mathcal{D} \subseteq \mathcal{D}^{\prime}$, then Problem 1 has a positive answer in this case. We also completely deal with Problem 1 if $\Gamma$ is cyclic.

Theorem 6.1. Suppose that $\Gamma$ is an l-group for some prime $l$ and that $\Pi$ is nilpotent. Then $\mathcal{D}^{\prime}(\Gamma, \Pi)=\mathcal{P}^{+}(\Gamma, \Pi)$.

Proof. Suppose that $\chi \in \mathcal{D}^{\prime}$. For each prime $p \neq l$, use Theorem 3.3 to write $\chi$ relative to $R\left(G_{p^{\prime}}\right) \otimes R\left(G_{p}\right)$ as $\chi=\sum_{\sigma_{p}} \xi_{\sigma_{p}} \otimes \operatorname{ind}_{\left[\sigma_{p}\right]}^{G_{p}} 1$. Since $\Gamma_{p}=1$, the only $\sigma_{p}: \Gamma_{p} \rightarrow \Pi_{p}$ in this sum is the trivial map; hence $\operatorname{ind}_{\left[\sigma_{p}\right]}^{G_{p}} 1$ is the character $\rho\left(G_{p}\right)$ of the regular representation and $\chi=\xi_{\sigma_{p}} \otimes \rho\left(G_{p}\right)$. In particular, $\chi$ vanishes off $G_{p^{\prime}}$. Varying $p \neq l$, it follows that $\chi$ vanishes off $G_{l}$.

Define the class function $\lambda$ on $G_{l}$ by $\lambda(g)=\chi(g) /\left|G_{l^{\prime}}\right|$. Then relative to $R(G)=$ $R\left(G_{l^{\prime}}\right) \otimes R\left(G_{l}\right)$ (actually with scalars extended to $\mathbf{Q}$ ) we have $\chi=\rho\left(G_{l^{\prime}}\right) \otimes \lambda$.

At the prime $l$ write $\chi=\sum_{\eta \in \operatorname{irr}\left(G_{l^{\prime}}\right)} \eta \otimes \chi_{\eta}$ with $\chi_{\eta} \in \mathcal{P}^{+}\left(\Gamma_{l}, \Pi_{l}\right)$. Since $\rho\left(G_{l^{\prime}}\right)=$ $\sum_{\eta \in \operatorname{irr}\left(G_{l^{\prime}}\right)} \eta(1) \eta$, we get

$$
\chi=\rho\left(G_{l^{\prime}}\right) \otimes \lambda=\sum_{\eta} \eta \otimes \eta(1) \lambda=\sum_{\eta} \eta \otimes \chi_{\eta},
$$

so we deduce that $\chi_{\eta}=\eta(1) \lambda$ for all $\eta \in \operatorname{irr}\left(G_{l^{\prime}}\right)$. Take $\eta$ to be the trivial character; then $\lambda=\chi_{1} \in \mathcal{P}^{+}\left(\Gamma_{l}, \Pi_{l}\right)$, and we can write $\lambda=\sum_{\sigma_{l}} a_{\sigma_{l}}$ ind $_{\left[\sigma_{l}\right]}^{G_{l}} 1$, where each $a_{\sigma_{l}}$ is a non-negative integer. 
Since $\rho\left(G_{l^{\prime}}\right) \otimes \operatorname{ind}_{\left[\sigma_{l}\right]}^{G_{l}} 1=\operatorname{ind}_{\left[\sigma_{l}\right]}^{G} 1$, we have

$$
\chi=\rho\left(G_{l^{\prime}}\right) \otimes \lambda=\sum_{\sigma_{l}} a_{\sigma_{l}} \rho\left(G_{l^{\prime}}\right) \otimes \operatorname{ind}_{\left[\sigma_{l}\right]}^{G_{l}} 1=\sum_{\sigma_{l}} a_{\sigma_{l}} \operatorname{ind}_{\left[\sigma_{l}\right]}^{G} 1 .
$$

Thus $\chi \in \mathcal{P}^{+}(\Gamma, \Pi)$, and the proof is complete.

Corollary 6.2. $\Gamma$ is a subgroup of $S G L_{n}(\mathbf{Z \Pi})$ if and only if $\Gamma$ is isomorphic to a subgroup of $\Pi^{n}$ (the direct product of $n$ copies of $\Pi$.)

Proof. Suppose that $\Gamma \subseteq S G L_{n}(\mathbf{Z \Pi})$. From Corollary 2.7, $\Gamma$ is nilpotent, so in order to prove that $\Gamma$ is a subgroup of $\Pi^{n}$ it suffices to prove that $\Gamma_{l} \subseteq \Pi^{n}$ for each prime $l$ dividing $\Gamma$. Hence we may assume that $\Gamma$ is an $l$-group. Then Theorem 6.1 implies that $\mathcal{D}(\Gamma, \Pi)=\mathcal{P}^{+}(\Gamma, \Pi)$, and then from Proposition $2.3, u \Gamma u^{-1} \subseteq \Pi^{n}$.

The converse is clear.

Theorem 6.3. Suppose that $\Pi$ is nilpotent. Then $\mathcal{D}=\mathcal{P}^{+}$for all cyclic $\Gamma$ if and only if $\Pi$ has at most one non-cyclic Sylow p-subgroup.

Proof. Suppose that $\Pi$ has at most one non-cyclic Sylow $p$-subgroup. We will show that $\mathcal{D}^{\prime}=\mathcal{P}^{+}$, and therefore that $\mathcal{D}=\mathcal{P}^{+}$. Fix a prime $p$, which exists by hypothesis, such that $\Pi_{p^{\prime}}$ is cyclic, and therefore has a faithful character $\lambda$ of degree 1. Let $\chi$ be in $\mathcal{D}^{\prime}$. Choose $\Sigma$ as in the proof of Proposition 4.3, namely

$$
\Sigma=\left\{\sigma \in \operatorname{hom}(\Gamma, \Pi): \sigma_{p} \in \Sigma_{p}\right\},
$$

where $\Sigma_{p} \subset \operatorname{hom}\left(\Gamma_{p}, \Pi_{p}\right)$ is a complete set of homomorphisms up to conjugacy in $\Pi_{p}$. By Proposition 4.3, $\chi \in \mathcal{P}$, and since $\Gamma$ is cyclic, we may write, by Lemma 4.2 ,

$$
\chi=\sum_{\sigma \in \Sigma} a_{\sigma} \operatorname{ind}_{[\sigma]}^{G} 1 \quad \text { for unique } a_{\sigma} \in \mathbf{Z} .
$$

We must show that $a_{\sigma} \geq 0$ for all $\sigma$.

Relative to $R(G)=R\left(G_{p^{\prime}}\right) \otimes R\left(G_{p}\right)$, we have $\operatorname{ind}_{[\sigma]}^{G} 1=\operatorname{ind}_{\left[\sigma_{p^{\prime}}\right]}^{G_{p^{\prime}}} 1 \otimes \operatorname{ind}_{\left[\sigma_{p}\right]}^{G_{p}} 1$, giving

$$
\chi=\sum_{\sigma} a_{\sigma} \operatorname{ind}_{\left[\sigma_{p^{\prime}}\right]}^{G_{p^{\prime}}} 1 \otimes \operatorname{ind}_{\left[\sigma_{p}\right]}^{G_{p}} 1 .
$$

From equation (3.2), we have

$$
\chi=\sum_{\sigma_{p} \in \Sigma_{p}} \xi_{\sigma_{p}} \otimes \operatorname{ind}_{\left[\sigma_{p}\right]}^{G_{p}} 1, \quad \xi_{\sigma_{p}} \in R^{+}\left(G_{p^{\prime}}\right) .
$$

Comparing these equations, using linear independence of $\left\{\operatorname{ind}_{[\sigma]}^{G} 1: \sigma \in \Sigma\right\}$ from Lemma 4.2 , we get

$$
\xi_{\sigma_{p}}=\sum_{\tau} a_{\tau} \operatorname{ind}_{\left[\tau_{p^{\prime}}\right]}^{G_{p^{\prime}}} 1 \in R^{+}\left(G_{p^{\prime}}\right)
$$

where the sum is over $\tau \in \Sigma$ such that $\tau_{p}=\sigma_{p}$. Then $\left\langle\xi_{\sigma_{p}}, \eta\right\rangle_{G_{p^{\prime}}} \geq 0$ for all irreducible characters $\eta$ of $G_{p^{\prime}}=\Gamma_{p^{\prime}} \times \Pi_{p^{\prime}}$, in particular for $\eta=\lambda^{*} \sigma_{p^{\prime}} \otimes \lambda$, where $\lambda^{*}$ is the contragredient of $\lambda$. But

$$
\begin{aligned}
\left\langle\xi_{\sigma_{p}}, \lambda^{*} \sigma_{p^{\prime}} \otimes \lambda\right\rangle & =\sum_{\tau} a_{\tau}\left\langle 1, \operatorname{res}_{\left[\tau_{p^{\prime}}\right]}^{G_{p^{\prime}}}\left(\lambda^{*} \sigma_{p^{\prime}} \otimes \lambda\right)\right\rangle_{\left[\tau_{p^{\prime}}\right]} \\
& =\sum_{\tau} a_{\tau} /\left|\Gamma_{p^{\prime}}\right| \sum_{\gamma \in \Gamma_{p^{\prime}}} \lambda\left(\sigma_{p^{\prime}} \gamma^{-1}\right) \lambda\left(\tau_{p^{\prime}} \gamma\right)=\sum_{\tau} a_{\tau}\left\langle\lambda \sigma_{p^{\prime}}, \lambda \tau_{p^{\prime}}\right\rangle_{\Gamma_{p^{\prime}}},
\end{aligned}
$$


and this equals $a_{\sigma}$ since $\lambda \sigma_{p^{\prime}}$ and $\lambda \tau_{p^{\prime}}$ are different irreducible characters of $\Gamma_{p^{\prime}}$ unless $\sigma_{p^{\prime}}=\tau_{p^{\prime}}$, that is, $\tau=\sigma$. Thus $a_{\sigma} \geq 0$.

Conversely, suppose that $\Pi$ has at least 2 non-cyclic Sylow $p$-subgroups. First suppose that $\Pi$ has a subgroup of the form $\Pi^{\prime}=C_{p_{1}} \times C_{p_{1}} \times C_{p_{2}} \times C_{p_{2}} \cong C_{p_{1} p_{2}} \times$ $C_{p_{1} p_{2}}$, where $p_{1}, p_{2}$ are distinct primes. Using the construction in Lemma 5.1 with $\Gamma=C_{p_{1} p_{2}}, G^{\prime}=\Gamma \times \Pi^{\prime}, N^{\prime}=1 \times \Pi^{\prime}$, there exists a $\mathbf{Z} G^{\prime}$-lattice $M^{\prime}$ satisfying (a) and (b) whose character is $\chi^{\prime}=-\operatorname{ind}_{[\tau]}^{G^{\prime}} 1+\sum_{\sigma \in \Sigma^{\prime}} \operatorname{ind}_{[\sigma]}^{G^{\prime}} 1$. By Lemma $2.4, \chi=\operatorname{ind}_{G^{\prime}}^{G} \chi^{\prime}$ is in $\mathcal{D}(\Gamma, \Pi)$. But $\chi=-\operatorname{ind}_{[\tau]}^{G} 1+\sum_{\sigma \in \Sigma^{\prime}}$ ind ${ }_{[\sigma]}^{G} 1$ is not in $\mathcal{P}^{+}$by Lemma 4.2, since $\tau=1$ is not $\Pi$-conjugate to an element of $\Sigma^{\prime}$. Thus $\mathcal{D}(\Gamma, \Pi) \neq \mathcal{P}^{+}(\Gamma, \Pi)$.

If $\Pi$ has at least 2 non-cyclic Sylow $p$-subgroups but does not have a subgroup isomorphic to $C_{p_{1}} \times C_{p_{1}} \times C_{p_{2}} \times C_{p_{2}}$, where $p_{1}, p_{2}$ are distinct primes, then $\Pi_{2}$ is a quaternion group, and $\Pi$ has a subgroup of the form $\Pi^{\prime}=C_{p} \times C_{p} \times Q_{8}$ where $p$ is an odd prime. Apply the construction of Lemma 5.2, where $\Gamma=C_{p} \times C_{4} \cong C_{4 p}$, $G^{\prime}=\Gamma \times \Pi^{\prime}, N^{\prime}=1 \times \Pi^{\prime}$, to get

$$
\chi^{\prime}=r\left(-\operatorname{ind}_{[\tau]}^{G^{\prime}} 1+\sum_{\sigma \in \Sigma^{\prime}} \operatorname{ind}_{[\sigma]}^{G^{\prime}} 1\right) \in \mathcal{D}\left(\Gamma, \Pi^{\prime}\right) \text { for some } r \geq 1
$$

As above, $\chi=\operatorname{ind}_{G^{\prime}}^{G} \chi^{\prime}$ is in $\mathcal{D}(\Gamma, \Pi)$ by Lemma 2.4 , but to show that $\chi \notin \mathcal{P}^{+}$we must be careful in our choice of $\tau$. Let $A$ be a cyclic normal subgroup of index 2 in $\Pi_{2}$ and choose $\tau$ whose image is $A \cap \Pi^{\prime}$. Then the construction of Lemma 5.2 applies, since $\operatorname{im} \tau$, which is normal in $\Pi$, is not $\Pi$-conjugate to any $\operatorname{im} \sigma$ with $\sigma \in \Sigma^{\prime}$. Applying Lemma 4.2 as before completes the proof.

\section{Finite Generation of $\mathcal{D}$}

Theorem 7.1. If $\Pi$ is nilpotent, then $\mathcal{D}^{\prime}$ and $\mathcal{D}$ are finitely generated semigroups.

Proof. Set

$$
X=\bigoplus_{H \leq G} \operatorname{ind}_{H}^{G} \mathbf{Z}, \quad \text { where } H \text { varies over all subgroups of } G .
$$

For each prime $p$ dividing $|G|$, enumerate the distinct non-isomorphic indecomposable summands of $\mathbf{Z}_{p} \otimes X$ : suppose they are $X(p, i), 1 \leq i \leq n_{p}$, and suppose that $X(p, i)$ affords the character $\chi(p, i)$ of $G$. Let $n=\sum n_{p}$, summed over primes dividing $|G|$. We shall use some ideas in the proof of a result of Jones [CR, 33.2]. Following some of the notation of $[\mathrm{CR}]$, let $C$ denote the additive semigroup of $n$-tuples of non-negative integers; partially order $C$ by writing $\left(a_{i}\right) \leq\left(b_{i}\right)$ in $C$ if $a_{i} \leq b_{i}$ for $1 \leq i \leq n$. If the $\mathbf{Z} G$-lattice $M$ satisfies (a) and ( $\left.\mathrm{b}^{\prime}\right)$, then by Proposition 3.4 and the Krull-Schmidt Theorem for $\mathbf{Z}_{p} G$-lattices, $\mathbf{Z}_{p} \otimes M$ can be written uniquely as a direct sum of modules $X(p, i)$ :

$$
\mathbf{Z}_{p} \otimes M \cong \bigoplus_{1 \leq i \leq n_{p}} X(p, i)^{m(p, i)}, \quad \text { for unique non-negative integers } m(p, i) .
$$

Let $\theta(M)$ denote the ordered $n$-tuple in $C$ whose entries are the integers $m(p, i)$. Let $\widehat{\mathcal{D}}^{\prime}$ be the set of $n$-tuples $\theta(M) \in C$ where $M$ ranges over all $\mathbf{Z} G$-lattices which satisfy (a) and $\left(\mathrm{b}^{\prime}\right)$; similarly, let $\widehat{\mathcal{D}}$ be the set of $\theta(M) \in C$ where $M$ satisfies (a) and (b). Given $\theta(M)=(a(p, i))$ in $\widehat{\mathcal{D}}$, we associate to $\theta(M)$ the character $\sum_{p} a(p, i) \chi(p, i)$; this gives us a mapping of $\widehat{\mathcal{D}}$ onto $\mathcal{D}$. Similarly we have a mapping 
of $\widehat{\mathcal{D}}^{\prime}$ onto $\mathcal{D}^{\prime}$. Thus the theorem will follow if we can prove finite generation of $\widehat{\mathcal{D}}^{\prime}$ and $\widehat{\mathcal{D}}$.

We first prove that $\widehat{\mathcal{D}}^{\prime}$ is finitely generated. From Step 3 in the proof of Jones' Theorem [CR, p. 689], any subset of $C$ has a finite set of minimal elements in the partial order we have given $C$. Let $S$ be the finite set of minimal elements of $\widehat{\mathcal{D}}^{\prime}-\{0\}$. We claim that this set $S$ generates $\widehat{\mathcal{D}}^{\prime}$. To prove this, let $\theta(M)$ be an element of $\widehat{\mathcal{D}}^{\prime}$; we shall show that $\theta(M)$ is a sum of elements of $S$. This is true if $\theta(M) \in S$, so assume that there is an element $s \in S$ which is strictly smaller than $\theta(M)$. Also assume that if $L$ is a $\mathbf{Z} G$-lattice such that $\theta(L) \in \widehat{\mathcal{D}}^{\prime}$ and which has smaller Z-rank than $M$, then $\theta(L)$ is a sum of elements of $S$. Suppose that $s=\theta\left(M^{\prime}\right)$. Then locally at each prime, $M^{\prime}$ is a direct summand of $M$, so [CR, 31.12], there is a lattice $M^{\prime \prime}$ in the same genus as $M^{\prime}$ such that $M \cong M^{\prime \prime} \oplus M_{0}$ for some $\mathbf{Z} G$-lattice $M_{0}$. Then $\theta\left(M^{\prime \prime}\right)=\theta\left(M^{\prime}\right)$, and $M_{0}$ satisfies (a) and $\left(\mathrm{b}^{\prime}\right)$. Moreover, by our assumption on lattices with ranks smaller than that of $M, \theta\left(M_{0}\right)$ is a sum of elements of $S$. Since $\theta(M)=s+\theta\left(M_{0}\right)$, then $\theta(M)$ is a sum of elements of $S$, as claimed.

We next show that $\widehat{\mathcal{D}}$ is finitely generated. Let $S_{0}$ be the set of $s \in S$ so that $s=\theta(M)$ for some $M$ such that $\operatorname{res}_{N} M$ is stably free but $s=\theta\left(M^{\prime}\right)$ for no $M^{\prime}$ such that $\operatorname{res}_{N} M^{\prime}$ is free. Let $r$ be as in Lemma 2.5. Set

$$
T=\left(\widehat{\mathcal{D}} \cap\left\{\sum_{s \in S} a_{s} s: 0 \leq a_{s} \leq r, s \in S\right\}\right) \cup\left\{(r+1) s: s \in S_{0}\right\} .
$$

Note that if res $_{N} M$ is stably free, then since $r+1 \geq 2$, res ${ }_{N} M^{r+1}$ is free by [CR, 41.20], so $T \subseteq \widehat{\mathcal{D}}$. We claim that $T$ generates $\widehat{\mathcal{D}}$. Suppose that $d=\theta(D) \in \widehat{\mathcal{D}}$. As above, we assume that if $\theta(L) \in \widehat{\mathcal{D}}$ and $L$ has smaller Z-rank than $D$, then $\theta(L)$ is a sum of elements of $T$. Since $\widehat{\mathcal{D}}^{\prime}$ is generated by $S$, we can write

$$
d=\sum_{s \in I} a_{s} s \text { for a subset } I \subseteq S \text { with } a_{s} \geq 1, s \in I .
$$

Write $a_{s}=b_{s}+r c_{s}$ where $1 \leq b_{s} \leq r$ and $c_{s} \geq 0$, and set

$$
e=\sum_{s \in I} b_{s} s, \quad f=r \sum_{s \in I} c_{s} s
$$

so $d=e+f$. We have $d \in \widehat{\mathcal{D}}$, and since $r \mathcal{D}^{\prime} \subseteq \mathcal{D}$, then $f \in \widehat{\mathcal{D}}$, so we can find $\mathbf{Z} G$ lattices $D$ and $F$ satisfying (a) and (b) with $d=\theta(D)$ and $f=\theta(F)$. Also, $e=\theta\left(E^{\prime}\right)$ for a lattice $E^{\prime}$ satisfying (a) and ( $\left.\mathrm{b}^{\prime}\right)$. Now $\theta(D)=\theta\left(E^{\prime} \oplus F\right)$, so $D$ and $E^{\prime} \oplus F$ are in the same genus, and locally for all $p, F$ is a direct summand of $D$.

We will apply a result of Roiter and Jacobinski [CR, 31.32]; we must check that every irreducible $\mathbf{Q} G$-composition factor of $\mathbf{Q} F$ occurs more often as a composition factor of $\mathbf{Q} D$. This is so because $d=e+f$ and $e=\sum_{s \in I} b_{s} s$, where $b_{s}>0$ for $s \in I$. Thus $D \cong E \oplus F$ for some $\mathbf{Z} G$-lattice $E$. Restricting to $N$, we see that $\operatorname{res}_{N} E$ is stably free. If it is actually free (so, in particular, if we have the Eichler condition for $\mathbf{Q} N)$, then $e=\theta(E) \in T$, and we are done.

From [CR, 41.20], if $\sum_{s \in I} b_{s}>1$, then $\operatorname{res}_{N} E$ is free; so we may assume that $\sum_{s \in I} b_{s}=1$. Thus $I$ contains a single element $s_{0}$, and $e=s_{0}$. If $s_{0} \notin S_{0}$, then writing $s_{0}=\theta\left(E^{\prime}\right)$ where $E^{\prime}$ satisfies (a) and (b), it follows that $D$ is in the same genus as $E^{\prime} \oplus F$; we replace $D$ by $E^{\prime} \oplus F$ and we are done as before. So we assume that $s_{0} \in S_{0}$. 
Suppose that $f=0$. Then $d=s_{0} \notin \widehat{\mathcal{D}}$. Hence $f \neq 0$. Then $c_{s} \geq 1$ for some $s \in I$; since $I=\left\{s_{0}\right\}$, then $f=c_{s_{0}} r s_{0}$ with $c_{s_{0}} \geq 1$. Then $e=(r+1) s_{0}+\left(c_{s_{0}}-1\right)\left(r s_{0}\right)$ with $(r+1) s_{0} \in T$ and $\left(c_{s_{0}}-1\right)\left(r s_{0}\right)=\theta(L) \in \widehat{\mathcal{D}}$, where $L$ has smaller rank than $D$. Thus $d$ is indeed a sum of elements of $T$, and the proof is complete.

\section{Complements}

Assume we have $\Gamma, \Pi$ and $G=\Gamma \times \Pi$ as above. As in the proof of Theorem 3.3, $\mathcal{G}$ is the Galois group of $\mathbf{Q}(\zeta)$ over $\mathbf{Q}$, where $\zeta$ be a primitive $|G|$-th root of unity, and $\mathcal{G}_{p}$ denotes the Galois group of $\mathbf{Q}_{p}(\zeta)$ over $\mathbf{Q}_{p}$. We identify $\mathcal{G}_{p}$ as a subgroup of $\mathcal{G}$, namely the decomposition group at any prime of $\mathbf{Q}(\zeta)$ above $p$. Let $\Sigma_{p}$ be a complete set of elements of hom $\left(\Gamma_{p}, \Pi_{p}\right)$ up to conjugation by $\Pi_{p}$. As in [WCr], define a label for $\chi$ to be a collection $\mathbf{b}=\left\{b_{p}\right\}$ of functions

$$
b_{p}: \Sigma_{p} \times \operatorname{irr}\left(G_{p^{\prime}}\right) \rightarrow \mathbf{Z}_{\geq 0},
$$

one for each prime $p$, so that on writing

$$
\chi=\sum_{\eta \in \operatorname{irr}\left(G_{p^{\prime}}\right)} \eta \otimes \lambda_{\eta} \text { relative to } R(G)=R\left(G_{p^{\prime}}\right) \otimes R\left(G_{p}\right)
$$

we have

$$
\begin{array}{ll}
(\mathrm{i})_{p} & \lambda_{\eta}=\sum_{\sigma_{p} \in \Sigma_{p}} b_{p}\left(\sigma_{p}, \eta\right) \operatorname{ind}_{\left[\sigma_{p}\right]}^{G_{p}} 1, \\
(\mathrm{ii})_{p} & b_{p}\left(\sigma_{p}, \eta^{\omega}\right)=b_{p}\left(\sigma_{p}, \eta\right) \text { for all } \omega \in \mathcal{G}_{p} .
\end{array}
$$

Theorem 8.1. Suppose that $\Gamma$ and $\Pi$ are nilpotent. Then labels for $\chi$ are in bijection with genera of $\mathbf{Z} G$-lattices with character $\chi$ which satisfy (a) and $\left(\mathrm{b}^{\prime}\right)$.

Proof. The proof comes from a closer look at the proof of Theorem 3.3. Suppose that $M$ is a $\mathbf{Z} G$-lattice which satisfies (a) and $\left(\mathrm{b}^{\prime}\right)$. From equation (3.1), we have

$$
\mathbf{z}_{p} \otimes M \cong \bigoplus_{\sigma_{p} \in \operatorname{hom}\left(\Gamma_{p}, \Pi_{p}\right)} X_{\sigma_{p}} \otimes \operatorname{ind}_{\left[\sigma_{p}\right]}^{G_{p}} \mathbf{z}_{p}
$$

Instead of summing over $\sigma_{p} \in \operatorname{hom}\left(\Gamma_{p}, \Pi_{p}\right)$, we may sum over $\Sigma_{p}$, because replacing $\sigma_{p}$ by a $\Pi_{p}$-conjugate gives a $G_{p}$-conjugate of $\left[\sigma_{p}\right]$, hence a module isomorphic to $\operatorname{ind}_{\left[\sigma_{p}\right]}^{G_{p}} \mathbf{Z}_{p}$. Then the different groups $\left[\sigma_{p}\right], \sigma_{p} \in \Sigma_{p}$, are the vertices of the summands of $\mathbf{Z}_{p} \otimes M$, so the modules $X_{\sigma_{p}}$ are unique up to isomorphism, and their characters $\xi_{\sigma_{p}}$ give the well-defined equation

$$
\chi=\sum_{\sigma_{p} \in \Sigma_{p}} \xi_{\sigma_{p}} \otimes \operatorname{ind}_{\left[\sigma_{p}\right]}^{G_{p}} 1, \quad \xi_{\sigma_{p}} \in R^{+}\left(G_{p^{\prime}}\right) .
$$

Then (ii) $)_{p}$ follows from equation (3.3) and (i) $p_{p}$ comes from equation (3.4). Since these depend only on $\mathbf{Z}_{p} \otimes M$, the same label would be attached to any lattice in the same genus as $M$.

Conversely, suppose that $\mathbf{b}$ is a label. From (ii) $)_{p}, b\left(\sigma_{p}, \eta\right)$ just depends on the $\mathcal{G}_{p^{-}}$-orbit $\mathcal{O}$ containing $\eta$. Then as in equation (3.6), we let $\xi_{\sigma_{p}}=\sum_{\mathcal{O}} b\left(\sigma_{p}, \mathcal{O}\right) \tau_{\mathcal{O}}$, and $\xi_{\sigma_{p}} \in R_{\mathbf{Q}_{p}}^{+}\left(G_{p^{\prime}}\right)$. The lattice

$$
M\left(b_{p}\right)=\bigoplus_{\sigma_{p}} L_{\sigma_{p}} \otimes_{\mathbf{z}_{p}} \operatorname{ind}_{\left[\sigma_{p}\right]}^{G_{p}} \mathbf{z}_{p}
$$


in equation (3.7) satisfies the local versions $\left(\mathrm{a}_{p}\right)$ and $\left(\mathrm{b}_{p}\right)$ of $(\mathrm{a})$ and $(\mathrm{b})$, and has character $\chi$ by $(\mathrm{i})_{p}$. Then the $\mathbf{Z} G$-lattice $M=M(\mathbf{b})$ at the end of the proof has $\mathbf{Z}_{p} \otimes M \cong M\left(b_{p}\right)$ for all $p$, hence it satisfies (a) and $\left(\mathrm{b}^{\prime}\right)$; the construction of $M$ depends on the identifications $\phi_{p}$, but the genus of $M$ is well-defined.

Corollary 8.2. If $\Gamma$ is cyclic, there is only one genus of $\mathbf{Z} G$-modules having a given character in $\mathcal{D}^{\prime}$.

Proof. By Lemma 4.2, for each $p$ the set $\left\{\operatorname{ind}_{\left[\sigma_{p}\right]}^{G_{p}} 1: \sigma_{p} \in \Sigma_{p}\right\}$ is linearly independent; hence there is only one solution to $(\mathrm{i})_{p}$, and only one label for a character $\chi$.

Remarks. Given $\chi \in \mathcal{D}^{\prime}$, we want to decide whether $\chi \in \mathcal{D}$. We begin by determining all labels $\mathbf{b}$ for $\chi$ : this is a purely character-theoretic problem. For each $\mathbf{b}$ one then constructs a lattice $M=M(\mathbf{b})$ in the genus of the label. Deciding whether the genus of $M$ contains an $M^{\prime}$ with res ${ }_{N} M^{\prime}$ stably free can then be approached by genus class group methods, generalizing Theorem 3 of [WCr]. Carrying this out is a long computation which will answer the existence question "Is $\chi$ in $\mathcal{D}$ ?" (at least when we have the Eichler condition for $\mathbf{Z} \Pi$ ). However, the construction of $\alpha: \Gamma \rightarrow S G L_{n}(\mathbf{Z} \Pi)$ with double-action character $\chi$ takes still more calculation.

Nevertheless this is how the first example of $\S 5$ was found. It is typical of such computations that once an $M$ satisfying (a) and (b) is found, it is simpler to describe it directly, as we have done in $\S 5$.

Finally there is the issue of finding $\chi \in \mathcal{D}^{\prime}$, no multiple of which is in $\mathcal{P}^{+}$, in the first place. This amounts to finding generators of $\mathcal{D}^{\prime}$, and this again is a problem of character theory, by Theorem 3.3. In examples we have considered, $\mathcal{D}^{\prime}$ has many generators, even when $\Gamma$ is cyclic. More exploration of this, perhaps by computer, is still needed.

\section{REFERENCES}

[B] Peter Blanchard, Exceptional group ring automorphisms I, II, Comm. Algebra 25 (1997), 2727-2733, 2735-2742. MR 98k:20007

[CR] C. W. Curtis and I. Reiner, Methods of Representation Theory, vol. II, John Wiley \& Sons, 1987. MR 88f:20002

[F] Walter Feit, The Representation Theory of Finite Groups, North-Holland, 1982. MR 83g:20001

[K] Lee Klingler, Construction of a counterexample to a conjecture of Zassenhaus, Comm. Algebra 19 (1991), 2303-2330. MR 92:20004

[MRSW] Z. Marciniak, J. Ritter, S. Sehgal, and A. Weiss, Torsion units in integral group rings of some metabelian groups II, J. Number Theory 25 (1987), 340-352. MR 88k:20019

[R] Irving Reiner, Maximal Orders, Academic Press, 1975. MR 52:13910

[RS] K. W. Roggenkamp and L. L. Scott, On a conjecture of Zassenhaus for finite group rings, manuscript, 1987.

[S] Sudarshan Sehgal, Units in Integral Group Rings, Longman, 1993. MR 94m:16039

[WAn] Alfred Weiss, Rigidity of p-adic p-torsion, Annals of Math. 127 (1988), 317-332. MR 89g:20010

[WCr] , Torsion units in integral group rings, J. Reine Angew. Math. 415 (1991), 175187. MR 92c:20009

Department of Mathematical Sciences, University of Alberta, Edmonton, Alberta, CAnada T6G 2G1

E-mail address: gcliff@math.ualberta.ca

E-mail address: aweiss@math.ualberta.ca 